\title{
SYMPLECTIC STRUCTURES FOR THE CUBIC SCHRÖDINGER EQUATION IN THE PERIODIC AND SCATTERING CASE.
}

\author{
K.L. VANINSKY
}

\begin{abstract}
We develop a unified approach for construction of symplectic forms for $1 \mathrm{D}$ integrable equations with the periodic and rapidly decaying initial data. As an example we consider the cubic nonlinear Schrödinger equation.
\end{abstract}

\section{Introduction}

1.1. General remarks. The main technical tool for the study of soliton systems is commutator formalism. All fashionable soliton systems like the Korteveg-de-Vriez equation (KdV), the cubic nonlinear Schrödinger equation (NLS), the sin-Gordon equation, the Toda lattice, etc; have such representation. Within the commutator formalism approach the dynamical system appears as a compatibility condition for an over-determined system of equations. As an example, we consider the NLS equation with repulsive nonlinearity ${ }^{1}$

$$
i \psi^{\bullet}=-\psi^{\prime \prime}+2|\psi|^{2} \psi,
$$

where $\psi(x, t)$ is a complex function of spatial variable $x$ and time $t$. The flow is a compatibility condition for the commutator

$$
\left[\partial_{t}-V_{3}, \partial_{x}-V_{2}\right]=0
$$

with

$$
V_{2}(x, t)=V=-\frac{i \lambda}{2} \sigma_{3}+Y_{0}=-\frac{i \lambda}{2}\left(\begin{array}{cc}
1 & 0 \\
0 & -1
\end{array}\right)+\left(\begin{array}{cc}
0 & \bar{\psi} \\
\psi & 0
\end{array}\right)
$$

and

$$
V_{3}(x, t)=\frac{\lambda^{2}}{2} i \sigma_{3}-\lambda Y_{0}+|\psi|^{2} i \sigma_{3}-i \sigma_{3} Y_{0}^{\prime}
$$

The corresponding auxiliary linear problem

$$
\left(\partial_{x}-V\right) \boldsymbol{f}=0, \quad \quad \boldsymbol{f}=\left(\begin{array}{c}
f_{1} \\
f_{2}
\end{array}\right)
$$

Key words and phrases. nonlinear Schrödinger, symplectic.

35Q53; 58B99. The work is partially supported by NSF grant DMS-9971834.

${ }^{1}$ Prime' ${ }^{\prime}$ signifies derivative in variable $x$ and dot $\bullet$ in time. 
can be written in the form of an eigenvalue problem for the Dirac operator

$$
\mathfrak{D} \boldsymbol{f}=\left[\left(\begin{array}{cc}
1 & 0 \\
0 & -1
\end{array}\right) i \partial_{x}+\left(\begin{array}{cc}
0 & -i \bar{\psi} \\
i \psi & 0
\end{array}\right)\right] \boldsymbol{f}=\frac{\lambda}{2} \boldsymbol{f} .
$$

Another important feature of soliton systems is the Hamiltonian formulation. Here we assume that the potential $\psi(x, t)$ is $2 l$-periodic: $\psi(x+2 l, t)=\psi(x, t)$. For instance, the NLS flow can be written as

$$
\psi^{\bullet}=\left\{\psi, H_{3}\right\},
$$

with Hamiltonian $H_{3}=\frac{1}{2} \int_{-l}^{l}\left|\psi^{\prime}\right|^{2}+|\psi|^{4} d x=$ energy and bracket

$$
\{A, B\}=2 i \int_{-l}^{l} \frac{\partial A}{\partial \bar{\psi}(x)} \frac{\partial B}{\partial \psi(x)}-\frac{\partial A}{\partial \psi(x)} \frac{\partial B}{\partial \bar{\psi}(x)} d x .
$$

The bracket is nondegenerate. The corresponding symplectic form (up to a scalar) is:

$$
\omega_{0}=2 i<\delta \bar{\psi} \wedge \delta \psi>, \quad<\bullet>=\frac{1}{2 l} \int_{-l}^{l} d x .
$$

A priori it is not clear why the dynamical system, which arises as a compatibility condition has a Hamiltonian formulation. To put it differently, is it possible to obtain Hamiltonian formalism from the spectral problem?

Here we would like to make some historical remarks. Originally, the Hamiltonian formulation of basic integrable models was found as an experimental fact. For the $\mathrm{KdV}$ equation the computation of symplectic structure in terms of the scattering data was performed by Faddeev and Zakharov, [5]. It involved some nontrivial identities for the products of solutions. Later Kulish and Reiman, [14], noted that all higher symplectic structures also can be written in terms of the scattering data. Again, they used the scheme of [5] and explicit calculations. Finally, we note that Zakharov and Manakov, [28], for the NLS equation adopted a different approach. Instead of the symplectic structure they worked with the corresponding Poisson bracket. Again, using explicit formulas for the product of solutions they computed the Poisson bracket between the coefficients of the scattering matrix. An appearance of explicit formulas that are the moving force of all these computations seems to be quite mysterious. This was already discussed in the literature [4], and described as a "computational miracle".

The standard assumption needed to carry out spectral analysis is that the potential either is periodic or has rapid decay at infinity. We refer to the latter case as scattering. Recently, in connection with the Seiberg-Witten theory [23, 24], Krichever and Phong, [13] developed a new approach for the construction of symplectic formalism. The latest exposition of their results can be found in [3]. The main idea of the Krichever-Phong approach is to introduce in a universal way the two-form on the space of auxiliary linear operators. This form is written in terms 
of the operator itself and its eigenfunctions. The goal of this paper is to review the Krichever-Phong approach in the case of 1D periodic NLS and to extend it to the scattering case. Within the unified approach, we reduce the number of formulas and eliminate unnecessary explicit computations. For instance computation of the symplectic form in terms of the spectral data (both in the periodic and the scattering case) becomes an application of the Cauchy residue theorem.

1.2. The periodic case. We assume that the potential is periodic with the period $2 l: \psi(x+2 l, t)=\psi(x, t)$. The Krichever-Phong formula, in the NLS context, takes the form

$$
\omega_{0}=\sum_{P_{ \pm}} \operatorname{res}<\boldsymbol{e}^{*} J \delta V \wedge \delta \boldsymbol{e}>d \lambda .
$$

This formula defines a closed 2-form $\omega_{0}$ on the space of operators $\partial_{x}-V(x, \lambda)$ with $2 l$ periodic potential. The set-up for this formula is broadly as follows ${ }^{2}$.

The eigenvalue problem

$$
\left[J \partial_{x}-J V(x, \lambda)\right] \boldsymbol{e}(x, \lambda)=0, \quad J=i \sigma_{2}=\left(\begin{array}{cc}
0 & 1 \\
-1 & 0
\end{array}\right)
$$

has special solutions, so-called Floquet solutions determined by the property $\boldsymbol{e}(x+$ $2 l, \lambda)=w(\lambda) \boldsymbol{e}(x, \lambda)$. The complex constant $w(\lambda)$ is called a Floquet multiplier. For each value of the spectral parameter $\lambda$ there are two linearly independent Floquet solutions and two distinct Floquet multipliers. These solutions and correspondingly multipliers become single-valued functions of a point on the two-sheeted covering of the plane of spectral parameter $\lambda$. The simple points of the periodic/antiperiodic spectrum of the eigenvalue problem constitute branching points of the cover. We assume that there is a finite number of simple points (so-called, finite gap potential).

This two sheeted covering constitutes a hyperelliptic Riemann surface $\Gamma$ with two infinities $P_{+}$and $P_{-}$(Fig. 1). Each point $Q=(\lambda, \pm)$ of $\Gamma$ is specified by the value of spectral parameter $\lambda$ and the sheet "+" or "-" which determines the Floquet multiplier $w(Q)$ corresponding to this $\lambda$. At every point of the curve we also have a Floquet solution $\boldsymbol{e}(x, Q)$ which becomes a function of the point $Q$ and satisfies the identity $\boldsymbol{e}(x+2 l, Q)=w(Q) \boldsymbol{e}(x, Q)$. The Floquet solution $\boldsymbol{e}(x, Q)$ has an exponential singularity at infinities and plays the role of so-called Baker-Akhiezer function for the curve $\Gamma$.

At every point of the curve $\Gamma$ we can define another solution $\boldsymbol{e}^{*}(x, Q)$. This is the Floquet solution which is brought from a point on the different sheet but with the same value of the spectral parameter $\lambda$. It is transposed and suitably normalized. The operator $J \partial_{x}-J V(x, \lambda)$ acts on the solution $\boldsymbol{e}^{*}(x, Q)$ as an adjoint i.e. on

\footnotetext{
${ }^{2}$ We refer to Section 2 for detailed discussion.
} 


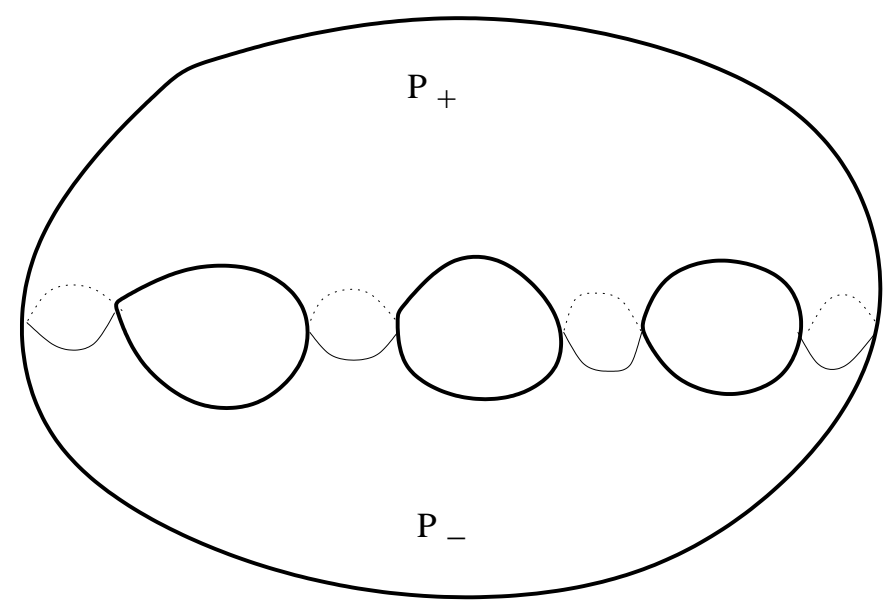

FiguRE 1. Smooth Riemann surface $\Gamma$.

the right:

$$
\boldsymbol{e}^{*}(x, Q)\left[J \partial_{x}-J V\right]=0 .
$$

It is assumed that the phase space consists of smooth $2 l$-periodic functions $\psi(x)$ or equivalently operators $\partial_{x}-V(x, \lambda)$ with $2 l$-periodic potential. The NLS flow acts on this space as it acts on the space of functions $\psi(x)$. All notions of differential geometry with obvious conventions can be applied to this space of operators. On the space of potentials we have a variation $\delta \psi(x)$. Thus for a fixed value of the spectral parameter $\lambda$ we have well defined variation $\delta V(x, \lambda)$. The variations $\delta \boldsymbol{e}(x, Q), \delta \boldsymbol{e}^{*}(x, Q)$ are defined correctly when $\lambda=\lambda(Q)$ is fixed. Therefore, at each point $Q$ of the surface $\Gamma$ we have well defined meromorphic in $Q$ the two-form

$$
<\boldsymbol{e}^{*} J \delta V \wedge \delta \boldsymbol{e}>d \lambda
$$

It takes values in the space of skew-symmetric two-forms on the space of operators $\partial_{x}-V$. The result of Krichever and Phong states that the sum of residues of this form at infinities $P_{ \pm}$is nothing but the symplectic form $\omega_{0}$.

The formula has a lot of good properties. First, it produces all higher symplectic structures by introducing the weight $\lambda^{n}$ under the residue

$$
\omega_{n}=\sum_{P_{ \pm}} \operatorname{res} \lambda^{n}<\boldsymbol{e}^{*} J \delta V \wedge \delta \boldsymbol{e}>d \lambda, \quad n=1,2, \ldots
$$

Second, it easily leads to the Darboux coordinates, or in physics terminology the separation variables, see Sklyanin [25]. These are local coordinates where the symplectic form $\omega_{0}$ takes the simple canonical form

$$
\omega_{0}=\frac{2}{i} \sum_{k} \delta p\left(\gamma_{k}\right) \wedge \delta \lambda\left(\gamma_{k}\right)
$$


This merits special explanation. It is well-known since the work of FlashkaMcLaughlin, [6], that the poles $\gamma_{k}$ of Floquet solutions lead to the Darboux coordinates for symplectic forms ${ }^{3}$. Recently, a lot of work was performed, [15], to construct such variables for the Ruijsenaars-Sneider and the Moser-Calogero systems. This required formidable technical machinery and extensive computations. At the same time, as it was demonstrated by Krichever, [11], the formula leads to the same result only by applying the Cauchy residue theorem.

1.3. The Scattering case. The main goal of the present paper is to show that suitably interpreted the new approach can be adopted for soliton systems with rapidly decaying initial data on the entire line. This is so-called the scattering case $^{4}$.

For such potentials one can define so-called Jost solutions $J_{ \pm}(x, \lambda)$. These are matrix solutions of the auxiliary linear problem $J_{ \pm}^{\prime}=V J_{ \pm}$with the asymptotics $J_{ \pm}^{\prime}(x, \lambda)=\exp \left(-i \frac{\lambda}{2} x \sigma_{3}\right)+o(1)$, as $x \rightarrow \pm \infty$. Their columns $J_{ \pm}=\left[\boldsymbol{j}_{ \pm}^{(1)}, \boldsymbol{j}_{ \pm}^{(2)}\right]$ are analytic in the corresponding upper/lower half-plane.

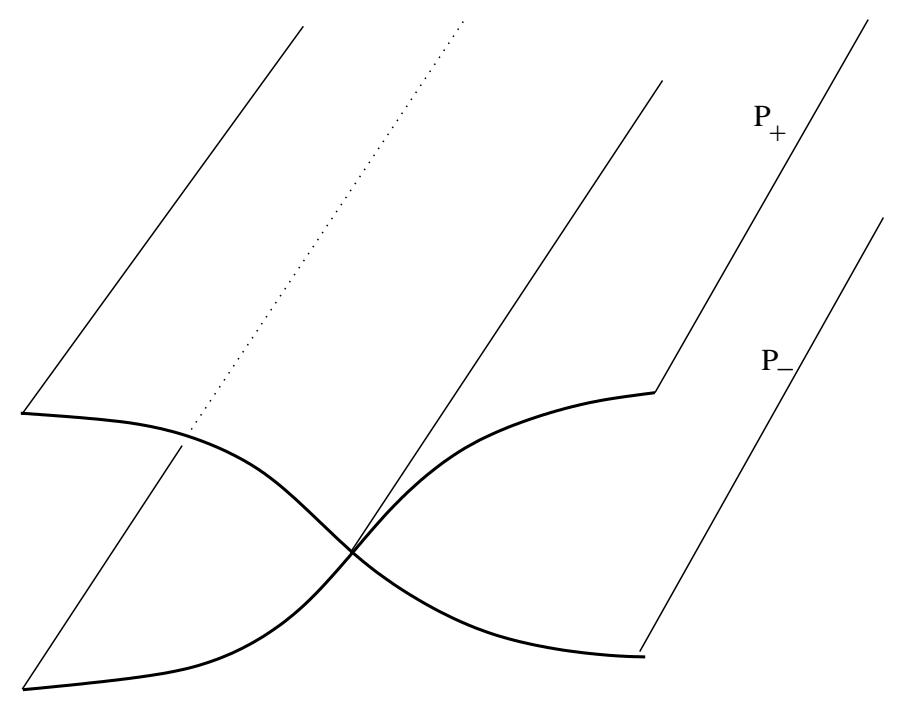

Figure 2. Singular Riemann surface $\Gamma_{\infty}$.

Our construction of the associated Riemann surface $\Gamma_{\infty}$ is a geometrical interpretation of what is called the Riemann-Hilbert approach to the scattering problem, see [4]. A singular curve $\Gamma_{\infty}$ is obtained by taking two copies of the complex plane and gluing them to each other along the real line (Fig. 2). The curve $\Gamma_{\infty}$ has two infinities $P_{+}$and $P_{-}$and continuum set of singular points above the real line. The

\footnotetext{
${ }^{3}$ See also Novikov-Veselov, [22], for general discussion.

${ }^{4}$ We refer to Section 3 for detailed definitions.
} 
standard Jost solutions are lifted on $\Gamma_{\infty}$ and become the single valued function of a point on the curve. Different branches of BA function are connected along the real line by the scattering matrix $S$ :

$$
S(\lambda)=\frac{1}{a}\left[\begin{array}{rr}
1 & \bar{b} \\
-b & 1
\end{array}\right] .
$$

The Jost solution has exponential singularity at infinities and plays the role of the Baker-Akhiezer function for the curve $\Gamma_{\infty}$. This construction is explained in detail in Section 3.

The formula of Theorem 3.4 looks similar to the periodic case

$$
\omega_{0}=\text { trace } \operatorname{res} \frac{1}{2}\left[<H_{+}^{*} J \delta V \wedge \delta H_{+}>+<H_{-}^{*} J \delta V \wedge \delta H_{-}>\right] d \lambda .
$$

The only difference now is that we work with the matrix solutions

$$
H_{+}(\lambda)=\left[\boldsymbol{j}_{-}^{(1)}(\lambda), \boldsymbol{j}_{+}^{(2)}(\lambda)\right] \quad \text { and } \quad H_{-}(\lambda)=\left[\boldsymbol{j}_{+}^{(1)}(\lambda), \boldsymbol{j}_{-}^{(2)}(\lambda)\right]
$$

and $H_{+}^{+}(\lambda)=\sigma_{1} H_{+}^{T}, H_{-}^{+}(\lambda)=\sigma_{1} H_{-}^{T}$, with

$$
\sigma_{1}=\left(\begin{array}{ll}
0 & 1 \\
1 & 0
\end{array}\right) .
$$

The averaging now corresponds to the integration on the entire line

$$
<\bullet>=\int_{-\infty}^{+\infty} d x .
$$

The residue can be computed explicitly $\omega_{0}=2 i<\delta \bar{\psi} \wedge \delta \psi>$. Theorem 3.6 states that the symplectic structure can be put in the Darboux form

$$
\omega_{0}=\frac{1}{\pi i} \int_{-\infty}^{+\infty} \frac{\delta \bar{b}(\lambda) \wedge \delta b(\lambda)}{|a(\lambda)|^{2}} d \lambda
$$

where $a$ and $b$ are coefficients of the scattering matrix $S$. Again identically to the periodic case this result is obtained by applying the Cauchy residue theorem. Only now the sum of the residues in the affine part of the curve is replaced by its' continuous analog. This is the integral which stays in the right hand side of the formula.

The unified approach to construction of symplectic forms produces an interesting problem. As we see, the symplectic form constructed in the periodic case has two systems of Darboux coordinates. One system is associated with poles of the Floquet solution. It is the divisor-quasimomentum Darboux coordinates. Another system of Darboux coordinates is the action-angle variables. At the same time in the scattering case we know only one system of Darboux coordinates. These are 
action-angle variables. What is the correct analog of the divisor-quasimomentum in the scattering case? This is a subject of future publication, [27].

We conclude this introduction by expressing thanks to A. Its, H. McKean and I. Krichever for stimulating discussions. We are also greatful to anonymous referee for remarks that helped to improve the presentation.

\section{The PERIOdic CASE.}

2.1. The Direct Spectral Problem. We provide here information needed in the next section for construction of symplectic forms. We refer to classical books $[20,21]$ for standard facts of spectral theory and algebraic-geometrical approach to solitons.

The NLS equation

$$
i \psi^{\bullet}=-\psi^{\prime \prime}+2|\psi|^{2} \psi
$$

where $\psi(x, t)$ is a smooth complex function $2 l$-periodic in $x$, is a Hamiltonian system

$$
\psi^{\bullet}=\{\psi, H\},
$$

with the Hamiltonian $H=\frac{1}{2} \int_{-l}^{l}\left|\psi^{\prime}\right|^{2}+|\psi|^{4} d x=$ energy and the bracket

$$
\{A, B\}=2 i \int_{-l}^{l} \frac{\partial A}{\partial \bar{\psi}(x)} \frac{\partial B}{\partial \psi(x)}-\frac{\partial A}{\partial \psi(x)} \frac{\partial B}{\partial \bar{\psi}(x)} d x .
$$

The NLS Hamiltonian $H=H_{3}$ is one in the infinite series of conserved integrals of motion.

$$
\begin{aligned}
& H_{1}=\frac{1}{2} \int_{-l}^{l}|\psi|^{2} d x, \\
& H_{2}=\frac{1}{2 i} \int_{-l}^{l} \psi \bar{\psi}^{\prime} d x, \\
& H_{3}=\frac{1}{2} \int_{-l}^{l}\left|\psi^{\prime}\right|^{2}+|\psi|^{4} d x, \quad \text { etc. }
\end{aligned}
$$

These Hamiltonians produce an infinite hierarchy of flows $e^{t X_{m}}, m=1,2, \ldots$.

The first in the hierarchy is the phase flow $e^{t X_{1}}$ generated by the vector field

$$
X_{1}: \quad \psi^{\bullet}=\left\{\psi, H_{1}\right\}=-i \psi .
$$

The phase flow is a compatibility condition for

$$
\left[\partial_{t}-V_{1}, \partial_{x}-V_{2}\right]=0
$$


with $^{5} V_{1}=\frac{i}{2} \sigma_{3}$ and

$$
V_{2}=-\frac{i \lambda}{2} \sigma_{3}+Y_{0}=\left(\begin{array}{cc}
-\frac{i \lambda}{2} & 0 \\
0 & \frac{i \lambda}{2}
\end{array}\right)+\left(\begin{array}{cc}
0 & \bar{\psi} \\
\psi & 0
\end{array}\right) .
$$

We often omit the subscript $V=V_{2}$. The second, translation flow $e^{t X_{2}}$ generated by

$$
X_{2}: \quad \psi^{\bullet}=\left\{\psi, H_{2}\right\}=\psi^{\prime}
$$

is equivalent to 2.2 with $V_{1}$ replaced by $V_{2}$. Finally, the third, original NLS flow 2.1 is a compatibility condition for 2.2 with $V_{1}$ replaced by

$$
V_{3}=\frac{\lambda^{2}}{2} i \sigma_{3}-\lambda Y_{0}+|\psi|^{2} i \sigma_{3}-i \sigma_{3} Y_{0}^{\prime}
$$

All flows of infinite hierarhy $e^{t X_{m}}, m=1,2, \ldots$ commute with each other

$$
\left[\partial_{\tau_{m}}-V_{m}, \partial_{\tau_{n}}-V_{n}\right]=0 .
$$

The first times $\tau_{1}, \tau_{2}$ and $\tau_{3}$ correspond to the first three flows.

We introduce a $2 \times 2$ transition matrix $M(x, y, \lambda), x \geq y$; that satisfies

$$
M^{\prime}(x, y, \lambda)=V(x, \lambda) M(x, y, \lambda), \quad M(y, y, \lambda)=I .
$$

The solution is given by the formula

$$
M(x, y, \lambda)=\exp \int_{y}^{x} V(\xi, \lambda) d \xi .
$$

The matrix $M(x, y, \lambda)$ is unimodular because $V$ is traceless.

The symmetry

$$
\sigma_{1} \bar{V}(x, \lambda) \sigma_{1}=V(x, \bar{\lambda})
$$

produces the same relation for the transition matrix

$$
\sigma_{1} \bar{M}(x, y, \lambda) \sigma_{1}=M(x, y, \bar{\lambda})
$$

Another symmetry

$$
V^{T}(x, \lambda) J=-J V(x, \lambda)
$$

where $J=i \sigma_{2}$, implies

$$
M^{T}(x, y, \lambda)^{-1} J=J M(x, y, \lambda) .
$$

The quantity $\Delta(\lambda)=\frac{1}{2} \operatorname{trace} M(l,-l, \lambda)$ is called a discriminant. The formula 2.3 implies $\bar{\Delta}(\lambda)=\Delta(\bar{\lambda})$ and $\Delta(\lambda)$ is real for real $\lambda$. The eigenvalues of the

\footnotetext{
${ }^{5}$ Here and below $\sigma$ denotes the Pauli matrices

$$
\sigma_{1}=\left(\begin{array}{cc}
0 & 1 \\
1 & 0
\end{array}\right), \quad \sigma_{2}=\left(\begin{array}{cc}
0 & -i \\
i & 0
\end{array}\right), \quad \sigma_{3}=\left(\begin{array}{cc}
1 & 0 \\
0 & -1
\end{array}\right) .
$$
}


monodromy matrix have a name of Floquet multipliers and they are roots of the quadratic equation

$$
w^{2}-2 \Delta w+1=0
$$

The Floquet multipliers are given by the formula $w=\Delta \pm \sqrt{\Delta^{2}-1}$. The values of $\lambda: w(\lambda)= \pm 1$ constitute the points of the periodic/antiperiodic spectrum. The corresponding auxiliary linear problem

$$
\left(\partial_{x}-V\right) \boldsymbol{f}=0, \quad \boldsymbol{f}^{T}=\left(f_{1}, f_{2}\right) ;
$$

can be written in the form of an eigenvalue problem for the self-adjoint Dirac operator

$$
\mathfrak{D} \boldsymbol{f}=\left[\left(\begin{array}{cc}
1 & 0 \\
0 & -1
\end{array}\right) i \partial_{x}+\left(\begin{array}{cc}
0 & -i \bar{\psi} \\
i \psi & 0
\end{array}\right)\right] \boldsymbol{f}=\frac{\lambda}{2} \boldsymbol{f}
$$

The self-adjointness implies that points of the spectra are real.

Example. Let $\psi \equiv 0$. The corresponding monodromy matrix can be easily computed $M(x, y, \lambda)=e^{-i \frac{\lambda}{2} \sigma_{3}(x-y)}$. We have $\Delta(\lambda)=\cos \lambda l$ and double eigenvalues at the points $\lambda_{n}^{ \pm}=\frac{\pi n}{l}$. If $\mathrm{n}$ is even/odd, then the corresponding $\lambda_{n}^{ \pm}$belongs to the periodic/ anti-periodic spectrum.

For a generic potential the double points $\lambda_{n}^{ \pm}$of the periodic/anti-periodic spectrum split, but they always stay real. The size of the spectral gap is determined, roughly speaking, by the corresponding Fourier coefficients of the potential. In our considerations we assume that there is a finite number of $g+1$ open gaps in the spectrum

$$
\ldots<\lambda_{n-1}^{-}=\lambda_{n-1}^{+}<\lambda_{n}^{-}<\lambda_{n}^{+}<\ldots<\lambda_{n+g}^{-}<\lambda_{n+g}^{+}<\lambda_{n+g+1}^{-}=\lambda_{n+g+1}^{+}<\ldots
$$

These are so-called finite gap potentials which are dense among all potentials.

The Floquet multipliers become single-valued on the Riemann surface:

$$
\Gamma=\left\{Q=(\lambda, w) \in \mathbb{C}^{2}: \quad R(\lambda, w)=\operatorname{det}[M(l,-l, \lambda)-w I]=0\right\} .
$$

The Riemann surface consists of two sheets covering the plane of the spectral parameter $\lambda$.

Example. Let $\psi \equiv 0$. We have $\Delta(\lambda)=\cos \lambda l$ and quadratic equation 2.5 has the solutions $w(\lambda)=e^{ \pm i l \lambda}$. The Riemann surface $\Gamma=\Gamma_{+}+\Gamma_{-}$is reducible and consists of two copies of the complex plane $\mathbb{C}$ that intersect each other at the points of the double spectrum $\lambda_{n}^{ \pm}$. Each part $\Gamma_{+}$or $\Gamma_{-}$contains the corresponding infinity $P_{+}$ or $P_{-}$. The Floquet multipliers are single valued on $\Gamma$ :

$$
\begin{array}{lll}
w(Q)=e^{+i \lambda l}, & & Q \in \Gamma_{+} ; \\
w(Q)=e^{-i \lambda l}, & & Q \in \Gamma_{-} .
\end{array}
$$

For a finite-gap potential the Riemann surface $\Gamma$ is irreducible. There are three types of important points on $\Gamma$. These are the singular points, the points above $\lambda=\infty$ and the branch points which we discuss now in detail. 
- The singular points are determined by the condition

$$
\partial_{\lambda} R(\lambda, w)=\partial_{w} R(\lambda, w)=0 .
$$

These are the points $\left(\lambda^{ \pm}, \pm 1\right)$ of the double spectrum. At these points two sheets of the curve intersect.

- There are two nonsingular points $P_{+}$and $P_{-}$above $\lambda=\infty$. At these points ${ }^{6}$

$$
\begin{aligned}
& w(Q)=e^{+i \lambda l}(1+O(1 / \lambda)), \quad Q \in\left(P_{+}\right) ; \\
& w(Q)=e^{-i \lambda l}(1+O(1 / \lambda)), \quad Q \in\left(P_{-}\right) \text {. }
\end{aligned}
$$

- The branch points are specified by the condition

$$
\partial_{w} R(\lambda, w)=0 .
$$

They are different from the singular points and correspond to the simple periodic/antiperiodic spectrum. We denote these points by $s_{k}^{ \pm}=\left(\lambda_{k}^{ \pm},(-1)^{k}\right)$, $k=n, \ldots, n+g$. There are $2(g+1)$ of them, each has a ramification index 2 .

The desingularized curve $\Gamma$ is biholomorphicaly equivalent to a hyperelliptic curve with branch points at the points of the simple spectrum. We also denote the hyperellitic curve by $\Gamma$. The Riemann-Hurwitz formula for the genus of $\Gamma$ implies

$$
\text { genus }=\frac{R}{2}-n+1
$$

where $R$ is a total ramification index and $n$ is the number of sheets. Each branch point has a ramification index 1 and therefore $R=2(g+1)$ and $n=2$. Therefore, the genus of $\Gamma$ is $g$, one off the number of open gaps in the spectrum.

Let $\epsilon_{ \pm}$be a holomorphic involution on the curve $\Gamma$ permuting sheets

$$
\epsilon_{ \pm}:(\lambda, w) \longrightarrow(\lambda, 1 / w) .
$$

The fixed points of $\epsilon_{ \pm}$are the branch points of $\Gamma$. The involution $\epsilon_{ \pm}$permutes infinities $\epsilon_{ \pm}: P_{-} \longrightarrow P_{+}$. Let us also define on $\Gamma$ an antiholomorphic involution

$$
\epsilon_{a}:(\lambda, w) \longrightarrow(\bar{\lambda}, \bar{w}) \text {. }
$$

The involution $\epsilon_{a}$ also permutes infinities and commutes with $\epsilon_{ \pm}$. Points of the curve above gaps $\left[\lambda_{n}^{-}, \lambda_{n}^{+}\right]$where $|\Delta(\lambda)| \geq 1$ form $g+1$ fixed "real" ovals of $\epsilon_{a}$. We call them $a$-periods.

The quasimomentum $p(Q)$ is a multivalued function on the curve $\Gamma$. It is introduced by the formula $w(Q)=e^{i p(Q) 2 l}$. Evidently, it is defined up to $\frac{\pi n}{l}$, where $n$ is an integer. The asymptotic expansion for $p(Q)$ at infinities can be easily computed

$$
\pm p(\lambda)=\frac{\lambda}{2}-p_{0}^{ \pm}-\frac{p_{1}}{\lambda}-\frac{p_{2}}{\lambda^{2}} \ldots, \quad Q \in\left(P_{ \pm}\right), \quad \lambda=\lambda(Q) ;
$$

\footnotetext{
${ }^{6}$ The notation $Q \in(P)$ means that the point $Q$ is in the vicinity of the point $P$.
} 
where $p_{0}^{ \pm}=\frac{\pi k_{ \pm}}{l}, k_{ \pm}$is an integer and

$$
p_{1}=\frac{1}{l} H_{1}, \quad p_{2}=\frac{1}{l} H_{2}, \quad p_{3}=\frac{1}{l} H_{3}, \quad \text { etc. }
$$

Moreover, the function $w(Q)+w\left(\epsilon_{ \pm} Q\right)$ does not depend on the sheet and is equal to $2 \Delta(\lambda)$. Thus $\Delta(\lambda(Q))=\cosh i p(Q) 2 l$ and the formula

$$
d p= \pm \frac{1}{i 2 l} d \cosh ^{-1} \Delta(\lambda)= \pm \frac{1}{i 2 l} \frac{\Delta^{\bullet}(\lambda) d \lambda}{\sqrt{\Delta^{2}-1}}
$$

implies that differential $d p$ is of the second kind with double poles at the infinities: $\pm d p=d\left(\frac{\lambda}{2}+O(1)\right)$. The same formula implies that the differential $d p$ is pure complex on the real ovals. At the same time, the condition $w\left(s_{k}^{-}\right)=w\left(s_{k}^{+}\right)$requires the increment $p\left(s_{k}^{+}\right)-p\left(s_{k}^{-}\right)$to be real. Therefore, $d p$ has zero $a$-periods

$$
\int_{a_{k}} d p=0
$$

Since the Floquet multiplies are single-valued on $\Gamma$ for the $b$-periods we have

$$
\int_{b_{k}} d p=\frac{\pi n_{b_{k}}}{l}, \quad \quad n_{b_{k}} \in \mathbb{Z}, \quad k=1, \ldots, g .
$$

These are so-called the periodicity conditions, [21].

The Floquet solution is the vector-function

$$
\boldsymbol{e}(x, Q)=\left[\begin{array}{l}
e^{1}(x, Q) \\
e^{2}(x, Q)
\end{array}\right]
$$

which is a solution of the auxiliary spectral problem $\boldsymbol{e}^{\prime}=V \boldsymbol{e}$ with the property

$$
\boldsymbol{e}(x+2 l, Q)=M(l,-l, \lambda) \boldsymbol{e}(x, Q)=w(Q) \boldsymbol{e}(x, Q)
$$

and normalized by the condition

$$
e^{1}(-l, Q)+e^{2}(-l, Q)=1 .
$$

Remark. If $\boldsymbol{f}(x, \lambda)$ is a solution of the auxiliary problem

$$
\left(\partial_{x}-V(x, \lambda)\right) \boldsymbol{f}=0
$$

corresponding to $\lambda$, then $\hat{\boldsymbol{f}}=\sigma_{1} \overline{\boldsymbol{f}}$ is a solution of $\left(\partial_{x}-V(x, \bar{\lambda})\right) \hat{\boldsymbol{f}}=0$ corresponding to $\bar{\lambda}$.

Example. Let $\psi=0$. The Floquet solution is given by the formula

$$
\begin{array}{ll}
\boldsymbol{e}(x, Q)=e^{+i \frac{\lambda}{2}(x+l)} \boldsymbol{e}_{0}=e^{+i \frac{\lambda}{2}(x+l)}\left[\begin{array}{l}
0 \\
1
\end{array}\right], & Q \in \Gamma_{+}, \\
\boldsymbol{e}(x, Q)=e^{-i \frac{\lambda}{2}(x+l)} \hat{\boldsymbol{e}}_{0}=e^{-i \frac{\lambda}{2}(x+l)}\left[\begin{array}{l}
1 \\
0
\end{array}\right], & Q \in \Gamma_{-} .
\end{array}
$$

It has no poles in the affine part of the curve. 
For a general finite gap potential the situation is more complicated.

Lemma 2.1. The Floquet solution satisfies the identity

$$
e\left(x, \epsilon_{a} Q\right)=\sigma_{1} \overline{\boldsymbol{e}(x, Q)} .
$$

The Floquet solution $\boldsymbol{e}(x, Q)$ has poles common for both components at the points

$$
\gamma_{1}, \gamma_{2}, \ldots, \gamma_{g+1}
$$

Projections of poles $\mu_{k}=\lambda\left(\gamma_{k}\right)$ are real. Each $\gamma_{k}$ lies on the real oval above the corresponding open gap $\left[\lambda_{k}^{-}, \lambda_{k}^{+}\right]$. Each component $e^{i}(x, Q)$ has $g+1$ zeros

$$
\sigma_{1}^{i}(x), \sigma_{2}^{i}(x), \ldots, \sigma_{g+1}^{i}(x) ; \quad i=1,2 .
$$

These zeros depend on the parameter $x$. In the vicinity of infinities the function $\boldsymbol{e}(x, Q)$ has the asymptotics

$$
\boldsymbol{e}(x, Q)=e^{ \pm i \frac{\lambda}{2}(x+l)}\left[\boldsymbol{e}_{0} / \hat{\boldsymbol{e}}_{0}+o(1)\right], \quad Q \in\left(P_{ \pm}\right)
$$

Before proceeding to the proof of the Lemma we note that the differential equation for the monodromy matrix

$$
M^{\prime}(x, y, \lambda)=\left[-\frac{i \lambda}{2} \sigma_{3}+Y_{0}\right] M(x, y, \lambda), \quad M(y, y, \lambda)=I,
$$

multiplied (gauged) on the left and right by the matrices

$$
C=\left(\begin{array}{cc}
1 & 1 \\
i & -i
\end{array}\right) \quad \text { and } \quad C^{-1}=\frac{1}{2}\left(\begin{array}{cc}
1 & -i \\
1 & i
\end{array}\right)
$$

transforms into

$$
M^{\mathbb{R}}(x, y, \lambda)=\left[-\frac{i \lambda}{2} \sigma_{2}+Y_{0}^{\mathbb{R}}\right] M^{\mathbb{R}}(x, y, \lambda), \quad M^{\mathbb{R}}(y, y, \lambda)=I
$$

with

$$
Y_{0}^{\mathbb{R}}=\left(\begin{array}{cc}
q & p \\
p & -q
\end{array}\right), \quad \psi=q+i p
$$

This is a real version of the eigenvalue problem which is more convenient in some situations, see [19]. The Floquet solution $\boldsymbol{e}^{\mathbb{R}}(x, Q)$ corresponding to the real version of the eigenvalue problem is related to $\boldsymbol{e}(x, Q)$ by the formula

$$
\boldsymbol{e}^{\mathbb{R}}(x, Q)=C \boldsymbol{e}(x, Q) .
$$

Therefore the result of the Lemma for $\boldsymbol{e}(x, Q)$ follows from the corresponding result for $\boldsymbol{e}^{\mathbb{R}}(x, Q)$ given in [19]. We prefer to give a direct proof, though the gauge transformation is behind all arguments.

Proof. The proof is based on the explicit formula for the Floquet solution. Let

$$
M(x,-l, \lambda)=\left[\begin{array}{cc}
m_{11} & m_{12} \\
m_{21} & m_{22} \\
12 &
\end{array}\right](x,-l, \lambda)
$$


and $M_{11}, M_{12}$, etc. be the elements of the matrix $M(l,-l, \lambda)$. The Floquet solution $\boldsymbol{e}(x, Q)$ is given by the formula

$$
\boldsymbol{e}(x, Q)=A(Q)\left[\begin{array}{l}
m_{11} \\
m_{21}
\end{array}\right](x,-l, \lambda)+(1-A(Q))\left[\begin{array}{l}
m_{12} \\
m_{22}
\end{array}\right](x,-l, \lambda),
$$

where $\lambda=\lambda(Q)$ and the coefficient $A(Q)$ is

$$
A(Q)=\frac{M_{12}}{M_{12}-M_{11}+w(Q)} \quad \text { or } \quad A(Q)=\frac{w(Q)-M_{22}}{M_{21}-M_{22}+w(Q)} .
$$

To prove the formula note that the Floquet solution is a linear combination of columns of the monodromy matrix $M(x,-l, \lambda)$ :

$$
\boldsymbol{e}(x, Q)=A(Q)\left[\begin{array}{l}
m_{11} \\
m_{21}
\end{array}\right](x,-l, \lambda)+A^{\prime}(Q)\left[\begin{array}{l}
m_{12} \\
m_{22}
\end{array}\right](x,-l, \lambda), \quad \lambda=\lambda(Q) .
$$

The normalization condition 2.10 implies $A^{\prime}(Q)=1-A(Q)$. At the same time the Floquet solution is an eigenvector of the monodromy matrix

$$
M(l,-l, \lambda)\left[\begin{array}{c}
A(Q) \\
1-A(Q)
\end{array}\right]=w(Q)\left[\begin{array}{c}
A(Q) \\
1-A(Q)
\end{array}\right]
$$

This leads to two equations

$$
M_{11} A(Q)+M_{12}(1-A(Q))=w(Q) A(Q)
$$

or

$$
M_{21} A(Q)+M_{22}(1-A(Q))=w(Q)(1-A(Q)) .
$$

Each equation implies the corresponding formula for $A(Q)$.

The formulas 2.3 and 2.12 imply

$$
1-A\left(\epsilon_{a} Q\right)=\overline{A(Q)} \text {. }
$$

This and 2.3, 2.11 imply the stated identity for the Floquet solution.

The relation $M^{\mathbb{R}}=C M C^{-1}$ implies

$$
\begin{aligned}
M^{\mathbb{R}} & =\left[\begin{array}{ll}
M_{11}^{\mathbb{R}} & M_{12}^{\mathbb{R}} \\
M_{21}^{\mathbb{R}} & M_{22}^{\mathbb{R}}
\end{array}\right] \\
& =\frac{1}{2}\left[\begin{array}{cc}
M_{11}+M_{12}+M_{21}+M_{22} & i\left(M_{12}+M_{22}-M_{11}-M_{21}\right) \\
i\left(M_{11}+M_{12}-M_{21}-M_{22}\right) & M_{11}+M_{22}-M_{12}-M_{21}
\end{array}\right] .
\end{aligned}
$$

Due to $2.3 M^{\mathbb{R}}(\lambda)$ is real for real $\lambda$. Consider the function $M_{12}^{\mathbb{R}}(\lambda)$ and look at the roots $\mu_{n}: M_{12}^{\mathbb{R}}\left(\mu_{n}\right)=0$. For $\psi \equiv 0$ we have $M_{12}^{\mathbb{R}}(\lambda)=-\sin \frac{\lambda l}{2}$ with roots at the points $\mu_{n}=\frac{2 \pi n}{l}, n \in \mathbb{Z}$. When we add the potential the roots $\mu_{n}$ move but stay real. They are caught by open gaps or match double periodic/antiperiodic spectrum. Indeed at $\mu_{n}$ the matrix $M^{\mathbb{R}}$ is lower triangular and real entries $M_{11}^{\mathbb{R}}$ and $M_{22}^{\mathbb{R}}$ coincide with Floquet multipliers. Since $M_{11}^{\mathbb{R}} M_{22}^{\mathbb{R}}\left(\mu_{n}\right)=1$ we have $\left|\Delta\left(\mu_{n}\right)\right|=$ $\frac{1}{2}\left|M_{11}^{\mathbb{R}}+M_{22}^{\mathbb{R}}\right| \geq 1$. 
The points of the divisor $\gamma_{k} \in \Gamma$ lie above the points $\mu_{k}$ on the sheet with $w(Q)=M_{22}^{\mathbb{R}}\left(\mu_{n}\right)$. At these points the denominator in 2.12

$$
M_{12}-M_{11}+w(Q) \quad \text { or } \quad M_{21}-M_{22}+w(Q)
$$

vanishes. Indeed from $M_{12}^{\mathbb{R}}\left(\mu_{n}\right)=0$ we have

$$
M_{12}-M_{11}\left(\mu_{n}\right)=M_{21}-M_{22}\left(\mu_{n}\right)
$$

and $w(Q)=M_{22}^{\mathbb{R}}\left(\mu_{n}\right)=M_{11}-M_{12}\left(\mu_{n}\right)=M_{22}-M_{21}\left(\mu_{n}\right)$. Moreover $M_{22} \neq$ $M_{22}^{\mathbb{R}}\left(\mu_{n}\right)$. These produce a pole of the Floquet solution when $\mu_{n}$ lies in the open gap. When $\mu_{n}$ is caught by the periodic/antiperiodic spectrum the matrix $M\left(l,-l, \mu_{n}\right)= \pm I$ and the zero of denominator is annihilated by the zero of numerator in 2.12 .

The asimptotics of the Floquet solution follows from the formula 2.11 and

$$
M(x, y, \lambda)=e^{-i \frac{\lambda}{2} \sigma_{3}(x-y)}+o(1), \quad \text { when } \quad \lambda \rightarrow \infty .
$$

The Floquet solution $\boldsymbol{e}(x, Q)$ near infinities can be expanded into the asymptotic series

$$
\begin{aligned}
& \boldsymbol{e}(x, Q)=e^{+i \frac{\lambda}{2}(x+l)} \sum_{s=0}^{\infty} \boldsymbol{e}_{s}(x) \lambda^{-s}=e^{+i \frac{\lambda}{2}(x+l)} \sum_{s=0}^{\infty}\left[\begin{array}{l}
b_{s} \\
d_{s}
\end{array}\right] \lambda^{-s}, \quad Q \in\left(P_{+}\right), \\
& \boldsymbol{e}(x, Q)=e^{-i \frac{\lambda}{2}(x+l)} \sum_{s=0}^{\infty} \hat{\boldsymbol{e}}_{s}(x) \lambda^{-s}=e^{-i \frac{\lambda}{2}(x+l)} \sum_{s=0}^{\infty}\left[\begin{array}{l}
\bar{d}_{s} \\
\bar{b}_{s}
\end{array}\right] \lambda^{-s}, \quad Q \in\left(P_{-}\right),
\end{aligned}
$$

and $b_{0}=0, \quad d_{0}=1$. The coefficients $b_{s}, d_{s}$ can be computed from the relation

$$
-\left[\begin{array}{l}
b_{s}^{\prime} \\
d_{s}^{\prime}
\end{array}\right]+Y_{0}\left[\begin{array}{l}
b_{s} \\
d_{s}
\end{array}\right]=\frac{i}{2}\left(I+\sigma_{3}\right)\left[\begin{array}{c}
b_{s+1} \\
d_{s+1}
\end{array}\right], \quad s=0,1, \ldots,
$$

due to the diagonal form of the matrix

$$
\frac{i}{2}\left(I+\sigma_{3}\right)=\left[\begin{array}{ll}
i & 0 \\
0 & 0
\end{array}\right] .
$$

Indeed, relation 2.13 leads to the identities

$$
-b_{s}^{\prime}+\bar{\psi} d_{s}=i b_{s+1}
$$

and

$$
-d_{s}^{\prime}+\psi b_{s}=0 \text {. }
$$

These are supplemented by the boundary condition

$$
b_{s}(x)+\left.d_{s}(x)\right|_{x=-l}=0, \quad s \geq 1 .
$$

When $s=0$ using $b_{0}=0, d_{0}=1$ from 2.14 we obtain

$$
b_{1}=-i \bar{\psi}(x) \text {. }
$$


The identities 2.15 and 2.16 imply

$$
d_{1}=i \bar{\psi}_{0}-i \int_{-l}^{x}|\psi|^{2} d x^{\prime}, \quad \psi_{0}=\psi(-l)
$$

Similar, we compute

$$
\begin{aligned}
& b_{2}=\bar{\psi}^{\prime}+\bar{\psi} \bar{\psi}_{0}-\bar{\psi} \int_{-l}^{x}|\psi|^{2} d x^{\prime} \\
& d_{2}=-\bar{\psi}_{0}^{\prime}-\bar{\psi}_{0}^{2}+\int_{-l}^{x}\left[\psi \bar{\psi}^{\prime}+|\psi|^{2} \bar{\psi}_{0}-|\psi|^{2} \int_{-l}^{x^{\prime}}|\psi|^{2} d x^{\prime \prime}\right] d x^{\prime} .
\end{aligned}
$$

These formulae will be used for explicit computation of symplectic forms.

The Floquet solution $\boldsymbol{e}(x, Q)$ satisfies the identity

$$
\left[J \partial_{x}-J V\right] \boldsymbol{e}(x, Q)=0, \quad J=i \sigma_{2}
$$

which is just another way to write the spectral problem. Let us define the dual Floquet solution $\boldsymbol{e}^{+}(x, Q)=\left[e^{1+}(x, Q), e^{2+}(x, Q)\right]$ at the point $Q$ as

$$
\boldsymbol{e}^{+}(x, Q)=\boldsymbol{e}\left(x, \epsilon_{ \pm} Q\right)^{T} .
$$

It can be verified by a direct computation that the dual Floquet solution $\boldsymbol{e}^{+}(x, Q)$ satisfies $^{7}$

$$
\boldsymbol{e}^{+}(x, Q)\left[J \partial_{x}-J V\right]=0 .
$$

The fact that the Wronskian $\boldsymbol{e}^{+}(x, Q) J \boldsymbol{e}(x, Q)$ does not depend on $x$ can be verified by differentiation. Introducing the function

$$
\Phi(Q)=\boldsymbol{e}^{+}(x, Q) J \boldsymbol{e}(x, Q)=<\boldsymbol{e}^{+}(x, Q) J \boldsymbol{e}(x, Q)>=\frac{1}{2 l} \int_{-l}^{l} \boldsymbol{e}^{+}(x, Q) J \boldsymbol{e}(x, Q) d x,
$$

we define another dual Floquet solution $\boldsymbol{e}^{*}(x, Q)$ by the formula

$$
\boldsymbol{e}^{*}(x, Q)=\frac{\boldsymbol{e}^{+}(x, Q)}{\Phi(Q)} .
$$

Evidently, $\boldsymbol{e}^{*}(x, Q) J \boldsymbol{e}(x, Q)=1$. The symmetry 2.4 produces an analog of monodromy property 2.9 for the function $\boldsymbol{e}^{*}$ :

$$
\boldsymbol{e}^{*}(x+2 l, Q)=\boldsymbol{e}^{*}(x, Q) J M^{-1}(l,-l, \lambda) J^{-1}=w^{-1}(Q) \boldsymbol{e}^{*}(x, Q) .
$$

\footnotetext{
${ }^{7}$ The action of the differential operator $D=\sum_{j=0}^{k} \omega_{j} \partial^{j}$ on the row vector $f^{+}$is defined as $f^{+} D=\sum_{j=0}^{k}(-\partial)^{j}\left(f^{+} \omega_{j}\right)$
} 
Lemma 2.2. The function $\boldsymbol{e}^{*}(x, Q)$ has simple poles at the branch points $s_{k}^{ \pm}$. It has fixed zeros at $\gamma_{1}, \ldots, \gamma_{g+1}$. The other zeros for each component of the vector function $\boldsymbol{e}^{*}$ lie on every real oval and depend on the parameter $x$. The function $\boldsymbol{e}^{*}$ has the asymptotics at infinities

$$
\boldsymbol{e}^{*}(x, Q)= \pm e^{\mp \frac{i \lambda}{2}(x+l)}\left[\hat{\boldsymbol{e}}_{0}^{T} / \boldsymbol{e}_{0}^{T}+o(1)\right], \quad Q \in\left(P_{+} / P_{-}\right) .
$$

Proof. The function $\Phi(Q)$ is meromorphic with $2(g+1)$ poles on both sheets above points $\mu_{n}$ lying in open gaps and $2(g+1)$ zeros at the branching points $s_{k}^{ \pm}$. At infinities it has the asymptotics

$$
\Phi(Q)= \pm 1+o(1), \quad Q \in\left(P_{ \pm}\right) .
$$

Now it is easy to prove properties of the function $e^{*}(x, Q)$. It has poles at the branch points $s_{k}^{ \pm}$which arise from zeros of $\Phi(Q)$. It has zeros at $\gamma_{1}, \ldots, \gamma_{g+1}$, the poles of $\Phi(Q)$. Other poles of $\Phi(Q)$ are annihilated by the poles of $\boldsymbol{e}^{+}(x, Q)$. Other $g+1$ zeros of $\boldsymbol{e}^{*}(x, Q)$ which depend on $x$ are produced by the corresponding zeros of $\boldsymbol{e}^{+}(x, Q)$.

The asymptotics follows from the asymptotics for $\boldsymbol{e}(x, Q)$ and $\Phi(Q)$.

Consider periodic variations of the matrix $V(x, \lambda): \tilde{V}=V+\delta V$. Then $\tilde{p}(Q)=$ $p(Q)+\delta p(Q)+\ldots$. We need a standard formula connecting the variations $\delta p(Q)$ and $\delta V$.

Lemma 2.3. The following identity holds

$$
i \delta p(Q)=<\boldsymbol{e}^{*}(x, Q) J \delta V \boldsymbol{e}(x, Q)>.
$$

Proof. Let $\tilde{\boldsymbol{e}}(x, Q)$ be a Floquet solution corresponding to the deformed potential $\tilde{V}$. From the definition

$$
\boldsymbol{e}^{+}(x, Q)\left(\left[J \partial_{x}-J \tilde{V}\right] \tilde{\boldsymbol{e}}(x, Q)\right)=0
$$

and

$$
\left(\boldsymbol{e}^{+}(x, Q)\left[J \partial_{x}-J V\right]\right) \tilde{\boldsymbol{e}}(x, Q)=0 .
$$

Subtracting one identity from another, we have

$$
\boldsymbol{e}^{+}\left(J \partial_{x} \tilde{\boldsymbol{e}}\right)-\left(\boldsymbol{e}^{+} J \partial_{x}\right) \tilde{\boldsymbol{e}}=\boldsymbol{e}^{+} J \tilde{V} \tilde{\boldsymbol{e}}-\boldsymbol{e}^{+} J V \tilde{\boldsymbol{e}}=\boldsymbol{e}^{+} J \delta V \tilde{\boldsymbol{e}} .
$$

Integrating both sides, we have

$$
\int_{-l}^{+l} \boldsymbol{e}^{+} J \tilde{\boldsymbol{e}}^{\prime}+\boldsymbol{e}^{+^{\prime}} J \tilde{\boldsymbol{e}} d x=\left.\boldsymbol{e}^{+} J \tilde{\boldsymbol{e}}\right|_{-l} ^{+l}
$$

Using the identities

$$
\boldsymbol{e}^{+}(l, Q)=e^{-i p(Q) 2 l} \boldsymbol{e}^{+}(-l, Q)
$$

and

$$
\tilde{\boldsymbol{e}}(l, Q)=e_{16}^{i \tilde{p}(Q) 2 l} \tilde{\boldsymbol{e}}^{+}(-l, Q)
$$


for the LHS, we have

$$
\left(e^{i \tilde{p}(Q) 2 l} e^{-i p(Q) 2 l}-1\right) \boldsymbol{e}^{+}(x, Q) J \tilde{\boldsymbol{e}}(x, Q)=i \delta p(Q) 2 l \Phi(Q)+\text { lower order terms. }
$$

The RHS is equal to

$$
2 l<\boldsymbol{e}^{+}(x, Q) J \delta V \boldsymbol{e}(x, Q)>+ \text { lower order terms. }
$$

Collecting leading terms, we obtain the stated identity.

Consider a real hyperelliptic spectral curve $\Gamma$ of finite genus corresponding to some periodic potential $\psi$. Let us introduce the Baker-Akhiezer function $\boldsymbol{e}(\tau, x, t, Q)$ which depends on three parameters (times) $\tau, x$ and $t$ and has the asymptotics at infinities

$$
\boldsymbol{e}(\tau, x, t, Q)=e^{ \pm i\left(-\frac{1}{2} \tau+\frac{\lambda}{2} x-\frac{\lambda^{2}}{2} t\right)} \times\left[\boldsymbol{e}_{0} / \hat{\boldsymbol{e}}_{0}+o(1)\right], \quad Q \in\left(P_{+} / P_{-}\right) .
$$

The BA function has poles at the points $\gamma^{\prime}$, located on the real ovals. These properties define the BA function uniquely. The BA function can be written explicitly in terms of theta-functions of the curve $\Gamma$, [10]. The BA function has Bloch property in $x$-variable $\boldsymbol{e}(\tau, l, t, Q)=w(Q) \boldsymbol{e}(\tau,-l, t, Q)$ and satisfies the identities

$$
\begin{aligned}
& {\left[J \partial_{\tau}-J V_{1}(\tau, x, t)\right] \boldsymbol{e}(\tau, x, t, Q)=0,} \\
& {\left[J \partial_{x}-J V_{2}(\tau, x, t)\right] \boldsymbol{e}(\tau, x, t, Q)=0,} \\
& {\left[J \partial_{t}-J V_{3}(\tau, x, t)\right] \boldsymbol{e}(\tau, x, t, Q)=0 .}
\end{aligned}
$$

The three matrices $V_{1}, V_{2}$ and $V_{3}$ are given at the beginning of this section.

Let us define the dual BA function $\boldsymbol{e}^{+}(\tau, x, t, Q)$ at the point $Q$ as

$$
\boldsymbol{e}^{+}(\tau, x, t, Q) \equiv \boldsymbol{e}\left(\tau, x, t, \epsilon_{ \pm} Q\right)^{T} \text {. }
$$

The identity $w(Q) w\left(\epsilon_{ \pm} Q\right)=1$ implies $\boldsymbol{e}^{+}(\tau, l, t, Q)=w(Q)^{-1} \boldsymbol{e}^{+}(\tau,-l, t, Q)$. The dual BA function $\boldsymbol{e}^{+}(\tau, x, t, Q)$ satisfies dual identities $\boldsymbol{e}^{+}(\tau, x, t, Q)\left[J \partial_{\tau}-J V_{1}\right]=$ 0 , etc.

2.2. Symplectic Structures. We assumed in the previous section that the phase space consists of smooth $2 l$-periodic functions $\psi(x)$. Instead we can change the language and think about the phase space as a space of operators $\partial_{x}-V_{2}$ with $2 l$-periodic potential. The flows of the NLS hierarchy act on this space as well they act on the space of functions $\psi$. All notions of differential geometry can be applied to this space of operators with obvious conventions.

Lemma 2.4. The formula

$$
\omega_{0}=\sum_{P_{ \pm}} \operatorname{res}<\boldsymbol{e}^{*} J \delta V \wedge \delta \boldsymbol{e}>d \lambda,
$$

defines a closed 2-form $\omega_{0}$ on the space of operators $\partial_{x}-V_{2}$ with periodic potential. The flows $e^{t X_{m}}, m=1,2, \ldots$ on the space of operators defined by the formula

$$
\left[\partial_{\tau_{m}}-V_{m}, \partial_{x}-V_{2}\right]=0 \text {, }
$$


are Hamiltonian with the symplectic structure $\omega_{0}$ and the Hamiltonian function $H_{m}$ (up to unessential constant factor).

Remark. The formula

$$
\omega_{n}=\sum_{P_{ \pm}} \operatorname{res} \lambda^{n}<\boldsymbol{e}^{*} J \delta V \wedge \delta \boldsymbol{e}>d \lambda, \quad n=0,1 \ldots,
$$

defines a closed 2-form $\omega_{n}$ on the space of operators $\partial_{x}-V_{2}$ with periodic potential which satisfy the constrains $H_{k}=$ const, $k=1, \ldots, n$; see for details [13].

Before proceeding to the proof of the Lemma, we compute the first two symplectic structures using the formula

$$
\omega_{n}=\sum_{P_{ \pm}} \operatorname{res} \frac{\lambda^{n}}{\Phi(Q)}<\boldsymbol{e}^{+} J \delta V \wedge \delta \boldsymbol{e}>d \lambda, \quad n=1,2 .
$$

The result is

$$
\begin{aligned}
& \omega_{0}=2 i<\delta \bar{\psi} \wedge \delta \psi> \\
& \omega_{1}=\omega_{1}=<\delta \psi \wedge \delta \bar{\psi}^{\prime}+\delta \bar{\psi} \wedge \delta \psi^{\prime}+2 \delta \partial^{-1}|\psi|^{2} \wedge \delta|\psi|^{2}>.
\end{aligned}
$$

subject to the constraint $H_{1}=$ const. We present the computaion divided in small steps.

Step 1. Identity $\Phi\left(\tau_{ \pm} Q\right)=-\Phi(Q)$ implies

$$
\begin{array}{lll}
\frac{1}{\Phi(Q)}=\phi_{0}+\frac{\phi_{1}}{\lambda}+\frac{\phi_{2}}{\lambda^{2}}+\cdots, & & Q \in\left(P_{+}\right), \\
\frac{1}{\Phi(Q)}=-\phi_{0}-\frac{\phi_{1}}{\lambda}-\frac{\phi_{2}}{\lambda^{2}}-\cdots, & & Q \in\left(P_{-}\right) .
\end{array}
$$

Using definition of $\boldsymbol{e}(x, Q)$ and $\boldsymbol{e}^{+}(x, Q)$ from previous section, we have

$$
\begin{aligned}
& \phi_{0}=1, \\
& \phi_{1}=-<d_{1}+\bar{d}_{1}>.
\end{aligned}
$$

Step 2. Near $P_{+}$we obtain

$$
<\boldsymbol{e}^{+} J \delta V \wedge \delta \boldsymbol{e}>=\frac{s_{1}}{\lambda}+\frac{s_{2}}{\lambda^{2}}+\frac{s_{3}}{\lambda^{3}}+\cdots,
$$

where

$$
\begin{aligned}
s_{1}= & <\hat{\boldsymbol{e}}_{0}^{T} J \delta V \wedge \delta \boldsymbol{e}_{1}> \\
s_{2}= & <\hat{\boldsymbol{e}}_{0}^{T} J \delta V \wedge \delta \boldsymbol{e}_{2}>+<\hat{\boldsymbol{e}}_{1}^{T} J \delta V \wedge \delta \boldsymbol{e}_{1}>.
\end{aligned}
$$

Similar, at $P_{-}$, we obtain

$$
<\boldsymbol{e}^{+} J \delta V \wedge \delta \boldsymbol{e}>-\frac{\bar{s}_{1}}{\lambda}-\frac{\bar{s}_{2}}{\lambda^{2}}-\frac{\bar{s}_{3}}{\lambda^{3}}-\cdots
$$


Using the expansion for $\Phi(Q)$ from Step 1, we derive

$$
\begin{aligned}
& \omega_{0}=\phi_{0}\left(s_{1}+\bar{s}_{1}\right), \\
& \omega_{1}=\phi_{0}\left(s_{2}+\bar{s}_{2}\right)+\phi_{1}\left(s_{1}+\bar{s}_{1}\right) .
\end{aligned}
$$

Step 3. Computing $s_{1}$, we have

$$
s_{1}=<\hat{\boldsymbol{e}}_{0}^{T} J \delta V \wedge \delta \boldsymbol{e}_{1}>=<\delta \psi \wedge \delta b_{1}>.
$$

Using the formula $b_{1}=-i \bar{\psi}$, we obtain $s_{1}=-i<\delta \psi \wedge \delta \bar{\psi}>$ and 2.18.

Step 4. The first term in the formula for $s_{2}$ produces

$$
<\hat{\boldsymbol{e}}_{0}^{T} J \delta V \wedge \delta \boldsymbol{e}_{2}>=<\delta \psi \wedge \delta b_{2}>\text {. }
$$

Using recurrence relation 2.14: $b_{2}=i b_{1}^{\prime}-i \bar{\psi} d_{1}$, we obtain

$$
<\delta \psi \wedge i \delta b_{1}^{\prime}-i \delta \bar{\psi} d_{1}-i \bar{\psi} \delta d_{1}>\text {. }
$$

The second term in the formula for $s_{2}$ produces

$$
<\hat{\boldsymbol{e}}_{1}^{T} J \delta V \wedge \delta \boldsymbol{e}_{1}>=<-\bar{b}_{1} \delta \bar{\psi} \wedge \delta d_{1}+\bar{d}_{1} \delta \psi \wedge \delta b_{1}>
$$

Finally,

$$
s_{2}=<\delta \psi \wedge \delta \bar{\psi}^{\prime}-i\left(d_{1}+\bar{d}_{1}\right) \delta \psi \wedge \delta \bar{\psi}-i \delta|\psi|^{2} \wedge \delta d_{1}>
$$

Using the formula for $s_{1}$,

$$
\omega_{1}=<\delta \psi \wedge \delta \bar{\psi}^{\prime}+\delta \bar{\psi} \wedge \delta \psi^{\prime}-i \delta|\psi|^{2} \wedge \delta\left(d_{1}-\bar{d}_{1}\right)>
$$

The constraint $H_{1}=$ const implies $\left\langle\delta|\psi|^{2}\right\rangle=0$ and using the explicit formula for $d_{1}$, we obtain 2.19.

Proof. Closeness of the form $\omega_{0}$ follows from the result of next Lemma or from explicit formula 2.18. For the second statement we present a complete proof only for $m=0$. Higher flows can be treated similarly. In order to prove that the first flow is Hamiltonian one has to establish, [1]:

$$
i_{\partial_{t}} \omega_{0}=-\delta 2 H_{1},
$$

where $i_{\partial_{t}}$ is the contraction operator produced by the vector field $X_{1}$. Using timedependent BA functions $i_{\partial_{t}} \delta \boldsymbol{e}=\boldsymbol{e}^{\bullet}, i_{\partial_{t}} \delta V=V^{\bullet}$ we have

$$
i_{\partial_{t}} \omega_{0}=\sum_{P_{ \pm}} \operatorname{res}<\boldsymbol{e}^{*} J V^{\bullet} \delta \boldsymbol{e}>d \lambda-\operatorname{res}<\boldsymbol{e}^{*} J \delta V \boldsymbol{e}^{\bullet}>d \lambda
$$

Let us compute the residue at $P_{+}$. From the computation preceding the proof

$$
\begin{array}{lll}
\frac{1}{\Phi(Q)}=\phi_{0}+\frac{\phi_{1}}{\lambda}+\ldots, & Q \in\left(P_{+}\right), \\
\frac{1}{\Phi(Q)}=-\phi_{0}-\frac{\phi_{1}}{\lambda}-\ldots, & & Q \in\left(P_{-}\right) .
\end{array}
$$


Then, using $V^{\bullet}=\left[\frac{i}{2} \sigma_{3}, V\right]=i \sigma_{3} V$, we obtain

$$
\text { res }<\boldsymbol{e}^{*} J V^{\bullet} \delta \boldsymbol{e}>d \lambda=i \phi_{0}<\hat{\boldsymbol{e}}_{0}^{T} J \sigma_{3} Y_{0} \delta \boldsymbol{e}_{1}>\text {. }
$$

Similarly, using $\boldsymbol{e}^{\bullet}=\frac{i}{2} \sigma_{3} \boldsymbol{e}$ we have:

$$
\begin{aligned}
\operatorname{res}<\boldsymbol{e}^{*} J \delta V \boldsymbol{e}^{\bullet}>d \lambda & =i 2 \phi_{1}<\hat{\boldsymbol{e}}_{0}^{T} J \delta Y_{0} \sigma_{3} \boldsymbol{e}_{0}> \\
& +i 2 \phi_{0}\left[<\hat{\boldsymbol{e}}_{0}^{T} J \delta Y_{0} \sigma_{3} \boldsymbol{e}_{1}>+<\hat{\boldsymbol{e}}_{1}^{T} J \delta Y_{0} \sigma_{3} \boldsymbol{e}_{0}>\right] .
\end{aligned}
$$

The first term vanishes and

$$
\begin{aligned}
i_{\partial_{t}} \operatorname{res}<\boldsymbol{e}^{*} J \delta V \wedge \delta \boldsymbol{e}>d \lambda & =i \phi_{0}<\hat{\boldsymbol{e}}_{0}^{T} J \sigma_{3} Y_{0} \delta \boldsymbol{e}_{1}> \\
& -\frac{i}{2} \phi_{0}\left[<\hat{\boldsymbol{e}}_{0}^{T} J \delta Y_{0} \sigma_{3} \boldsymbol{e}_{1}>+<\hat{\boldsymbol{e}}_{1}^{T} J \delta Y_{0} \sigma_{3} \boldsymbol{e}_{0}>\right] .
\end{aligned}
$$

Similarly at $P_{-}$

$$
\begin{aligned}
i_{\partial_{t}} \operatorname{res}<\boldsymbol{e}^{*} J \delta V \wedge \delta \boldsymbol{e}>d \lambda & =-i \phi_{0}<\boldsymbol{e}_{0}^{T} J \sigma_{3} Y_{0} \delta \hat{\boldsymbol{e}}_{1}> \\
& +\frac{i}{2} \phi_{0}\left[<\boldsymbol{e}_{0}^{T} J \delta Y_{0} \sigma_{3} \hat{\boldsymbol{e}}_{1}>+<\boldsymbol{e}_{1}^{T} J \delta Y_{0} \sigma_{3} \hat{\boldsymbol{e}}_{0}>\right] .
\end{aligned}
$$

Finally, we obtain

$$
i_{\partial_{t}} \omega_{0}=i \phi_{0}\left[<\hat{\boldsymbol{e}}_{0}^{T} J \sigma_{3} Y_{0} \delta \boldsymbol{e}_{1}>-<\boldsymbol{e}_{0}^{T} J \sigma_{3} Y_{0} \delta \hat{\boldsymbol{e}}_{1}>\right]=-\delta 2 H_{1}
$$

2.3. Darboux coordinates. The formulas 2.18-2.19 give examples of symplectic forms. All these forms can be put in the Darboux form in the coordinates associated with poles of the Baker-Akhiezer function.

Lemma 2.5. The formula

$$
\xi_{0}(Q)=<\boldsymbol{e}^{*} J \delta V \wedge \delta \boldsymbol{e}>d \lambda
$$

defines meromorphic in $Q$ differential 2-form on $\Gamma$ with poles at $\gamma_{1}, \ldots, \gamma_{g+1}$ and $P_{+}, P_{-}$. The symplectic 2-form defined by the formula

$$
\omega_{0}=\sum_{P_{ \pm}} \operatorname{res} \xi_{0}(Q)
$$

can be written as

$$
\omega_{0}=\frac{2}{i} \sum_{k=1}^{g+1} \delta p\left(\gamma_{k}\right) \wedge \delta \lambda\left(\gamma_{k}\right) .
$$

Remark 1. The meaning of the right-hand side of this formula is the following. The curve $\Gamma$ (or its cover $\hat{\Gamma}$ ) is equipped with two meromorphic functions $\lambda(Q)$ and $p(Q)$. Their variation $\delta p(Q)$ and $\delta \lambda(Q)$ at the points of the divisor is computed for variation of the potential $\psi(x), \bar{\psi}(x) ;-l \leq x \leq l$. The RHS of the formula is the sum of an exterior products of these variations. 
Remark 2. In fact for a general smooth potential the divisor $\lambda\left(\gamma_{k}\right)$ and values of the quasimomentum $\cosh ^{-1} \Delta\left(\lambda\left(\gamma_{k}\right)\right)$ with suitably chosen sign (=sheet) determine the potential. In other words they are global coordinates on the phase space. First note that the discriminant $\Delta$ can be reconstructed from this data using Shannon interpolation, see [19]. Thus the curve $\Gamma$ is known. The potential can be effectively recovered from the divisor via trace formulas, see [18].

Proof. Note $\delta V=\delta Y_{0}$ does not depend on $\lambda$. Essential singularity of the Floquet solutions at $P_{ \pm}$cancels out, and infinities are simple poles for the form $\xi_{0}(Q)$. In the finite part of the curve $\xi_{0}(Q)$ has two sets of poles. One is the poles $\gamma_{1}, \ldots, \gamma_{g+1}$ of the Baker-Akhiezer function. Another is the branch points of the curve $\Gamma$. Вy the Cauchy theorem

$$
\sum_{P_{ \pm}} \operatorname{res} \xi_{0}(Q)=-\sum_{\gamma_{k}} \operatorname{res} \xi_{0}(Q)-\sum_{s_{k}} \operatorname{res} \xi_{0}(Q)
$$

Let us compute contribution of the first set of poles. Near $\gamma_{k}$ we have

$$
\boldsymbol{e}=\frac{\mathrm{res} \boldsymbol{e}}{\lambda-\lambda\left(\gamma_{k}\right)}+O(1)
$$

Therefore,

$$
\delta \boldsymbol{e}=\frac{\operatorname{res} \boldsymbol{e}}{\left(\lambda-\lambda\left(\gamma_{k}\right)\right)^{2}} \delta \lambda\left(\gamma_{k}\right)+O\left(\frac{1}{\lambda-\lambda\left(\gamma_{k}\right)}\right)
$$

and

$$
\delta \boldsymbol{e}=\frac{\boldsymbol{e}}{\lambda-\lambda\left(\gamma_{k}\right)} \delta \lambda\left(\gamma_{k}\right)+O\left(\frac{1}{\lambda-\lambda\left(\gamma_{k}\right)}\right) .
$$

Note that $\boldsymbol{e}^{*}\left(x, \gamma_{k}\right) \equiv 0$ and from Lemma 2.3 we obtain

$$
\operatorname{res}_{\gamma_{k}} \xi_{0}(Q)=<\boldsymbol{e}^{*} J \delta V \boldsymbol{e}>\left(\gamma_{k}\right) \wedge \delta \lambda\left(\gamma_{k}\right) \operatorname{res}_{\gamma_{k}}\left[\frac{d \lambda}{\lambda-\lambda\left(\gamma_{k}\right)}\right]=i \delta p\left(\gamma_{k}\right) \wedge \delta \lambda\left(\gamma_{k}\right) .
$$

Now consider branch points of the curve. They produce a nontrivial contribution, though the pole of $\boldsymbol{e}^{*}$ at the branch point is annihilated by the zero of the differential $d \lambda$. Nevertheless, the variation $\delta \boldsymbol{e}(x, Q)$ has a simple pole at $s_{k}$. First, let us make a general remark.

Consider, the variation of a function $f(Q, \psi, \bar{\psi})$ under variation of the potential $\psi(x), \bar{\psi}(x),-l \leq x \leq l$; taken for $Q$ in the vicinity of the branch point $s_{k}$ and a fixed value of $\lambda$. Such variation will have a pole at the branch point itself. At the branch point $\lambda$ fails to be a local parameter, but $w$ is fine due to the fact $\left.\partial_{\lambda} R(\lambda, w)\right|_{s_{k}} \neq 0$. Now, consider a function $f(Q, \psi, \bar{\psi})=f(w, \psi, \bar{\psi})$, and define its variation $\delta_{0}$ for a fixed value of $w$. Then,

$$
\delta f=\delta_{0} f+\frac{d f}{d w} \delta w
$$


Take, for example $f(Q)=\lambda(Q)$, then

$$
0=\delta_{0} \lambda+\frac{d \lambda}{d w} \delta w
$$

Therefore, for a general $f$ we have

$$
\delta f=\delta_{0} f-\frac{d f}{d \lambda} \delta_{0} \lambda=-\frac{d f}{d \lambda} \delta_{0} \lambda+O(1)
$$

The zero of the differential $d \lambda$ at the branch point produces the pole of $\delta f$.

We can proceed to the computation of the residues of $\xi_{0}(Q)$ at $s_{k}$. In the local parameter $\left(\lambda-\lambda\left(s_{k}\right)\right)^{1 / 2} \sim w-1, \lambda=\lambda(Q)$ :

$$
\delta \boldsymbol{e}(x, Q)=-\frac{\boldsymbol{e}_{1}(x)}{2} \frac{\delta \lambda\left(s_{k}\right)}{\left(\lambda-\lambda\left(s_{k}\right)\right)^{1 / 2}}+\ldots,
$$

where

$$
\boldsymbol{e}(x, Q)=\boldsymbol{e}_{0}(x)+\boldsymbol{e}_{1}(x)\left(\lambda-\lambda\left(s_{k}\right)\right)^{1 / 2}+\ldots, \quad Q \in\left(s_{k}\right) .
$$

Similarly,

and we have

$$
d \boldsymbol{e}(x, Q)=\frac{\boldsymbol{e}_{1}(x)}{2} \frac{d \lambda}{\left(\lambda-\lambda\left(s_{k}\right)\right)^{1 / 2}}+\ldots,
$$

$$
\delta \boldsymbol{e}(x, Q)=-\frac{d \boldsymbol{e}(x, Q)}{d \lambda} \delta \lambda\left(s_{k}\right)+O(1) .
$$

The leading term is the same as in general formula 2.22. Therefore, 2.21 implies $\operatorname{res}_{s_{k}} \xi_{0}(Q)=-\operatorname{res}_{s_{k}}\left[<\boldsymbol{e}^{*} J \delta V d \boldsymbol{e}>\right] \wedge \delta \lambda\left(s_{k}\right)=\operatorname{res}_{s_{k}}\left[<\boldsymbol{e}^{*} J \delta V d \boldsymbol{e}>\wedge \frac{d \lambda \delta w}{d w}\right]$.

Now using

$$
\begin{aligned}
\boldsymbol{e}^{*}(x, Q) & =\boldsymbol{e}^{*}(l, Q) M^{T}(l, x, \lambda)^{-1} \\
d \boldsymbol{e}(x, Q) & =d M(x,-l, \lambda) \boldsymbol{e}(-l, Q)+M(x,-l, \lambda) d \boldsymbol{e}(-l, Q)
\end{aligned}
$$

with the help $\left.d M(x, y, \lambda)\right|_{s_{k}}=0$ we obtain,

$\operatorname{res}_{s_{k}} \xi_{0}(Q)=\operatorname{res}_{s_{k}}\left[e^{*}(l, Q)<M^{T}(l, x, \lambda)^{-1} J \delta V M(x,-l, \lambda)>d \boldsymbol{e}(-l, Q) \wedge \frac{d \lambda \delta w}{d w}\right]$.

Symmetry 2.4 of the monodromy matrix implies

$$
<M^{T}(l, x, \lambda)^{-1} J \delta V M(x,-l, \lambda)>=\frac{1}{2 l} J \delta M(l,-l, \lambda),
$$

and using skew-symmetry of the wedge product

$$
\begin{aligned}
\operatorname{res}_{s_{k}} \xi_{0}(Q) & =\frac{1}{2 l} \operatorname{res}_{s_{k}}\left[\boldsymbol{e}^{*}(l, Q) J \delta M(l,-l, \lambda) d \boldsymbol{e}(-l, Q) \wedge \frac{d \lambda \delta w}{d w}\right] \\
& =\frac{1}{2 l} \operatorname{res}_{s_{k}}\left[\boldsymbol{e}^{*}(l, Q) J(\delta M(l,-l, \lambda)-\delta w) d \boldsymbol{e}(-l, Q) \wedge \frac{d \lambda \delta w}{d w}\right] .
\end{aligned}
$$


Identities 2.9 and 2.17 imply

$$
\begin{aligned}
\boldsymbol{e}^{*} J(\delta M-\delta w) & =\delta \boldsymbol{e}^{*} J(w-M), \\
J(w-M) d \boldsymbol{e} & =J(d M-d w) \boldsymbol{e} .
\end{aligned}
$$

Therefore,

$$
\operatorname{res}_{s_{k}} \xi_{0}(Q)=\frac{1}{2 l} \operatorname{res}_{s_{k}}\left[\delta \boldsymbol{e}^{*}(l) J(d M(l,-l, \lambda)-d w) \boldsymbol{e}(-l) \wedge \frac{d \lambda \delta w}{d w}\right] .
$$

Since $\boldsymbol{e}^{*}(l) J \boldsymbol{e}(-l)=w^{-1}$, we have

$$
\operatorname{res}_{s_{k}} \xi_{0}(Q)=\frac{1}{2 l} \operatorname{res}_{s_{k}}\left[\boldsymbol{e}^{*}(l) J \delta \boldsymbol{e}(-l) \wedge \delta w d \lambda\right] .
$$

The one form

$$
\boldsymbol{e}^{*}(l, Q) J \delta \boldsymbol{e}(-l, Q) \wedge \delta w(Q) d \lambda(Q)
$$

is holomorphic (in the parameter $\lambda$ ) outside of the poles $\gamma_{k}$ and the branch points. At infinity the essential singularity cancels out and due to 2.6-2.7, Lemmas 2.1 and 2.2

This implies

$$
e^{*}(l, Q) J \delta \boldsymbol{e}(-l, Q) \wedge \delta w(Q)=o\left(\frac{1}{\lambda}\right) .
$$

$$
\operatorname{res}_{P_{ \pm}}\left[\boldsymbol{e}^{*}(l, Q) J \delta \boldsymbol{e}(-l, Q) \wedge \delta w(Q) d \lambda(Q)\right]=0 .
$$

By the Cauchy theorem,

$$
\sum_{s_{k}} \operatorname{res}_{s_{k}}\left[\boldsymbol{e}^{*}(l) J \delta \boldsymbol{e}(-l) \wedge d \lambda \delta w\right]=-\sum_{\gamma_{k}} \operatorname{res}_{\gamma_{k}}\left[\boldsymbol{e}^{*}(l) J \delta \boldsymbol{e}(-l) \wedge d \lambda \delta w\right]
$$

Therefore, using $\boldsymbol{e}^{*}(l)=w^{-1} \boldsymbol{e}^{*}(-l)$, we have

$$
\begin{aligned}
\sum_{s_{k}} \operatorname{res}_{s_{k}} \xi_{0}(Q) & =-\frac{1}{2 l} \sum_{\gamma_{k}} \operatorname{res}_{\gamma_{k}}\left[\boldsymbol{e}^{*}(l) J \delta \boldsymbol{e}(-l) \wedge d \lambda \delta w\right] \\
& =-\frac{1}{2 l} \sum_{\gamma_{k}} \operatorname{res}_{\gamma_{k}}\left[\boldsymbol{e}^{*}(-l) J \delta \boldsymbol{e}(-l) \wedge d \lambda \frac{\delta w}{w}\right]
\end{aligned}
$$

Using the formula 2.20 we finally obtain

$$
\begin{aligned}
\sum_{s_{k}} \operatorname{res}_{s_{k}} \xi_{0}(Q) & =\sum_{\gamma_{k}} i \delta p\left(\gamma_{k}\right) \wedge \operatorname{res}_{\gamma_{k}}\left[\boldsymbol{e}^{*}(-l) J \delta \boldsymbol{e}(-l) d \lambda\right] \\
& =\sum_{\gamma_{k}} i \delta p\left(\gamma_{k}\right) \wedge \delta \lambda\left(\gamma_{k}\right) \operatorname{res}_{\gamma_{k}}\left[\frac{d \lambda}{\lambda-\lambda\left(\gamma_{k}\right)}\right] \\
& =\sum_{\gamma_{k}} i \delta p\left(\gamma_{k}\right) \wedge \delta \lambda\left(\gamma_{k}\right) .
\end{aligned}
$$


Remark. For the higher symplectic structures an analogous result holds

$$
\omega_{n}=\frac{2}{i} \sum_{k=1}^{g+1} \lambda^{n} \delta p\left(\gamma_{k}\right) \wedge \delta \lambda\left(\gamma_{k}\right), \quad n=0,1 \ldots ;
$$

subject to the constrains $H_{k}=$ const $, k=1, \ldots, n$.

2.4. Action-angle variables. Here we describe briefly another system of Darboux coordinates. We refer to the paper [19] for details.

The actions $I_{k}, k=1, \ldots, g+1$; are defined by the formula

$$
I_{k}=\frac{1}{4 \pi} \int_{a_{k}} p(\lambda) d \lambda .
$$

In the formula above the multivalued function $p(\lambda)$ is normalized such that $p\left(s_{k}\right)=$ $0, k=1, \ldots, g+1$. The angles $\theta_{k}, n=1, \ldots, g+1$; are

$$
\theta_{k}=\sum_{n=1}^{g+1} \int_{s_{n}}^{\gamma_{n}} \alpha_{k} .
$$

The differentials $\alpha_{k}, n=1, \ldots, g+1$; are of the third kind with poles at the infinities $P_{ \pm}$normalized in such a way that ${ }^{8}$

$$
\int_{a_{k}} \alpha_{n}=2 \pi \delta_{n}^{k}
$$

As it is proved in [19] by a direct computation

$$
\left\{\theta_{n}, I_{k}\right\}=\delta_{n}^{k}
$$

All other brackets vanish

$$
\left\{I_{n}, I_{k}\right\}=0, \quad\left\{\theta_{n}, \theta_{k}\right\}=0 .
$$

These formulas imply the identity for symplectic forms.

$$
\omega_{0}=2 \sum_{n=1}^{g+1} \delta I_{n} \wedge \delta \theta_{n} .
$$

We conclude this section with a few remarks.

Remark 1. Another way to prove the identity for symplectic forms without employing the Poisson bracket is found by Krichever, [11].

Remark 2. McKean, [17], proved various identities for 1-forms.

Remark 3. In the finite-gap case the curve $\Gamma$ is specified by $2(g+1)$ branch points. The actions $I_{k}, k=1, \ldots, g+1$; together with the $g$ other periods (see 2.8) of the differential $d p$ and the constant $p_{0}^{+}$determine the curve. This fact is due to Krichever, [12] (see also [2]). In the infinite-gap case it is shown in [19] that $I-\theta$ 's

\footnotetext{
${ }^{8} \delta_{n}^{k}$ is Kronecker delta.
} 
are global coordinates on the phase space for a general square integrable potential. This property holds for a finite gap potential as well.

\section{The Scattering Case}

3.1. Jost solutions. In the next sections we consider the scattering problem for the Dirac operator on the entire line with rapidly decaying potential. The Riemann-Hilbert approach to the scattering theory for canonical systems with summable potential was constructed by M.G. Krein and P.E. Melik-Adamian, $[8,9,16]$. This approach was used many times in soliton theory, [4].

To simplify the estimates we assume that the potential $\psi$ is from the Schwartz' space $S(\mathbb{R})$ of complex rapidly decreasing infinitely differentiable functions on the line such that

$$
\sup _{x}\left|\left(1+x^{2}\right)^{n} \psi^{(m)}(x)\right|<\infty \quad m, n=0,1, \ldots
$$

Let us introduce the reduced transition matrix $T(x, y, \lambda), x \geq y$; by the formula

$$
T(x, y, \lambda)=E^{-1}\left(\frac{\lambda x}{2}\right) M(x, y, \lambda) E^{-1}\left(-\frac{\lambda y}{2}\right),
$$

where $E\left(\frac{\lambda x}{2}\right)=\exp \left(-\frac{i \lambda x}{2} \sigma_{3}\right)$ is a solution of the free equation $(\psi \equiv 0)$. The matrix $T(x, y, \lambda)$ solves the equation

$$
T^{\prime}(x, y, \lambda)=Y_{0}(x) E(\lambda x) T(x, y, \lambda), \quad T(y, y, \lambda)=I .
$$

The spectral parameter enters multiplicatively into the RHS of the differential equation. The solution is given by the formula

$$
T(x, y, \lambda)=\exp \int_{y}^{x} Y_{0}(\xi) E(\lambda \xi) d \xi .
$$

The symmetry of the matrix $Y_{0}: \sigma_{1} Y_{0}(x) \sigma_{1}=\overline{Y_{0}(x)}$ is inherited by unimodular matrix T:

$$
\sigma_{1} T(x, y, \bar{\lambda}) \sigma_{1}=\overline{T(x, y, \lambda)} .
$$

For real $\lambda$ the formula 3.2 and the rapid decay of the potential imply an existence of the limit

$$
T(\lambda)=\lim T(x, y, \lambda)=\left(\begin{array}{cc}
a(\lambda) & \bar{b}(\lambda) \\
b(\lambda) & \bar{a}(\lambda)
\end{array}\right), \quad \text { when } \quad y \rightarrow-\infty \quad \text { and } \quad x \rightarrow+\infty
$$

and $|a(\lambda)|^{2}-|b(\lambda)|^{2}=1$. When the potential $\psi \in S(\mathbb{R})$, we have $b(\lambda) \in S(\mathbb{R})$.

We introduce Jost solutions $J_{ \pm}(x, \lambda)$ as a matrix solutions of the differential equation

$$
J_{ \pm}^{\prime}(x, \lambda)=V(x, \lambda) J_{ \pm}(x, \lambda), \quad J_{ \pm}(x, \lambda)=E\left(\frac{\lambda x}{2}\right)+o(1), \quad \text { when } \quad x \rightarrow \pm \infty .
$$


An existence and analytic properties of the Jost solutions follow from the integral representations

$$
\begin{aligned}
& J_{+}(x, \lambda)=E\left(\frac{x \lambda}{2}\right)+\int_{x}^{+\infty} \Gamma_{+}(x, \xi) E\left(\frac{\lambda \xi}{2}\right) d \xi \\
& J_{-}(x, \lambda)=E\left(\frac{x \lambda}{2}\right)+\int_{-\infty}^{x} \Gamma_{-}(x, \xi) E\left(\frac{\lambda \xi}{2}\right) d \xi
\end{aligned}
$$

The kernels $\Gamma_{ \pm}$are unique and infinitely smooth in both variables. Introducing the notation $J_{ \pm}=\left[\boldsymbol{j}_{ \pm}^{(1)}, \boldsymbol{j}_{ \pm}^{(2)}\right]$ we see from the integral representations that $\boldsymbol{j}_{-}^{(1)}(x, \lambda)$, $\boldsymbol{j}_{+}^{(2)}(x, \lambda)$ are analytic in $\lambda$ in the upper half-plane and continuous up to the boundary. Also, the columns $\boldsymbol{j}_{-}^{(2)}(x, \lambda), \boldsymbol{j}_{+}^{(1)}(x, \lambda)$ are analytic in the lower half-plane and continuous up to the boundary.

Now we describe analytic properties of the coefficient $a(\lambda)$ of the matrix $T(\lambda)$. The monodromy matrix $M(x, y, \lambda)$ can be written in the form

$$
M(x, y, \lambda)=J_{+}(x) J_{+}^{-1}(y)=J_{-}(x) J_{-}^{-1}(y) .
$$

Therefore,

$$
J_{+}^{-1}(x) M(x, y, \lambda) J_{-}(y)=J_{+}^{-1}(y) J_{-}(y)=J_{+}^{-1}(x) J_{-}(x) .
$$

The variables $x$ and $y$ separate and the above expression does not depend on $x$ or $y$ at all. By passing to the limit with $x \rightarrow+\infty, y \rightarrow-\infty$ we have

$$
T(\lambda)=J_{+}^{-1}(y) J_{-}(y)=J_{+}^{-1}(x) J_{-}(x) .
$$

Therefore,

$$
a(\lambda)=\boldsymbol{j}_{-}^{(1)^{T}}(\lambda) J \boldsymbol{j}_{+}^{(2)}(\lambda) .
$$

The properties of Jost solutions imply that

- $a(\lambda)$ is analytic in the upper half-plane and continuous up to the boundary;

- $a(\lambda)$ is root-free;

- $|a(\lambda)| \geq 1$ and $|a(\lambda)|^{2}-1 \in S(\mathbb{R})$ for $\lambda$ real, $a(\lambda)=1+o(1)$ as $|\lambda| \longrightarrow \infty$.

This coefficient will be used to construct the scattering curve $\Gamma_{\infty}$.

Let $p_{\infty}(\lambda)$ be such that $a(\lambda)=\exp \left(-i 2 p_{\infty}(\lambda)\right)$ for $\lambda$ in the upper half-plane. The quantity $p_{\infty}(\lambda)$ in analogous to the quasimomentum studied in the periodic case, see $[26]^{9}$. From the properties of $a(\lambda)$ it follow that $p_{\infty}(\lambda)$ is analytic in the upper half-plane and continuous up to the boundary; $\Im p_{\infty}(\lambda) \geq 0$ for $\Im \lambda \geq 0 ; p_{\infty}(\lambda)=$ $o(1)$ for $|\lambda| \rightarrow \infty$; for real $\lambda$, the density of the measure $d \mu_{\infty}(\lambda)=\Im p_{\infty}(\lambda) d \lambda$ belongs to $S(\mathbb{R})$. The function $p_{\infty}(\lambda)$ can be written in the form

$$
p_{\infty}(\lambda)=\frac{1}{\pi} \int \frac{d \mu_{\infty}(t)}{t-\lambda}
$$

\footnotetext{
${ }^{9}$ The spectral parameter $\lambda$ in the paper [26] has different scaling and should be replaced by $\frac{\lambda}{2}$. 
Expanding the denominator in inverse powers of $\lambda$, we obtain:

$$
p_{\infty}(\lambda)=-\sum_{k=0}^{\infty} \frac{1}{\lambda^{k+1}} \frac{1}{\pi} \int_{-\infty}^{+\infty} t^{k} d \mu_{\infty}(t)=-\frac{H_{1}}{\lambda}-\frac{H_{2}}{\lambda^{2}}-\frac{H_{3}}{\lambda^{3}}+\ldots
$$

where $H_{1}, H_{2}$ and $H_{3}$ are the integrals introduced above with $l=+\infty$. The expansion has an asymptotic character for $\lambda: \delta \leq \arg \lambda \leq \pi-\delta, \delta>0$.

To describe the asymptotic behavior in $x$ of the Jost solutions $\boldsymbol{j}_{+}^{(2)}(x, \lambda)$ and $\boldsymbol{j}_{-}^{(1)}(x, \lambda)$ we assume that $\lambda$ is real and fixed. Then,

$$
\begin{array}{ccc} 
& x \rightarrow-\infty & x \rightarrow+\infty \\
\boldsymbol{j}_{+}^{(2)} & a(\lambda) \boldsymbol{f}_{\rightarrow}(x, \lambda)-\bar{b}(\lambda) \boldsymbol{f}_{\leftarrow}(x, \lambda) & \boldsymbol{f}_{\rightarrow}(x, \lambda) \\
\boldsymbol{j}_{-}^{(1)} & \boldsymbol{f}_{\leftarrow}(x, \lambda) & a(\lambda) \boldsymbol{f}_{\leftarrow}(x, \lambda)+b(\lambda) \boldsymbol{f}_{\rightarrow}(x, \lambda),
\end{array}
$$

where

$$
\boldsymbol{f}_{\leftarrow}(x, \lambda)=\left[\begin{array}{c}
e^{-i \frac{\lambda}{2} x} \\
0
\end{array}\right], \quad \boldsymbol{f}_{\rightarrow}(x, \lambda)=\left[\begin{array}{c}
0 \\
e^{i \frac{\lambda}{2} x}
\end{array}\right]
$$

are solutions of the free equation.

We sketch the derivation of the asymptotics for $j_{+}^{(2)}(x, \lambda)$, when $x \rightarrow-\infty$. Let $\psi$ be a potential that vanishes outside the segment $[-L,+L]$. In this case formula 3.2 becomes

$$
T(\lambda)=\exp \int_{-L}^{L} Y_{0}(\xi) E(\lambda \xi) d \xi
$$

and $T(\lambda)$ is an entire unimodular function of $\lambda$ of the form

$$
T(\lambda)=\left[\begin{array}{ll}
a(\lambda) & \bar{b}(\bar{\lambda}) \\
b(\lambda) & \bar{a}(\bar{\lambda})
\end{array}\right]
$$

From the definition of the matrix $M$ :

$$
\boldsymbol{j}_{+}^{(2)}(L, \lambda)=M(L,-L, \lambda) \boldsymbol{j}_{+}^{(2)}(-L, \lambda),
$$

and from 3.1

$$
M(L,-L, \lambda)=\left[\begin{array}{cc}
a(\lambda) e^{-i \lambda L} & \bar{b}(\bar{\lambda}) \\
b(\lambda) & \bar{a}(\bar{\lambda}) e^{i \lambda L}
\end{array}\right] .
$$

Obviously, $\boldsymbol{j}_{+}^{(2)}(L, \lambda)=\boldsymbol{f}_{\rightarrow}(L, \lambda)$ and $\boldsymbol{j}_{+}^{(2)}(-L, \lambda)=c_{1} \boldsymbol{f}_{\rightarrow}(-L, \lambda)+c_{2} \boldsymbol{f}_{\leftarrow}(-L, \lambda)$ with unknown coefficients $c_{1}$ and $c_{2}$. Formula 3.4 for real $\lambda$ leads to the linear system

$$
\begin{aligned}
& c_{1} \bar{b}(\lambda)+c_{2} a(\lambda)=0 \\
& c_{1} \bar{a}(\lambda)+c_{2} b(\lambda)=1 .
\end{aligned}
$$

Solving for $c$ 's we obtain the stated formula. For a potential with noncompact support one has to take $L$ sufficiently large to make the error negligible.

The Riemann surface $\Gamma_{\infty}$ is obtained by gluing together along the real line two copies of the complex plane (see Figure 2). One copy we call "+" and another "-". 
Each copy has an infinity $P_{+}$or $P_{-}$. The point $Q \in \Gamma_{\infty}$ is determined by $\lambda=\lambda(Q)$ and specification of the sheet $Q=(\lambda, \pm)$. Let us define for the "+" copy $\boldsymbol{j}(x, Q)$ to be $\boldsymbol{j}_{+}^{(2)}(x, \lambda)$ if $\Im \lambda>0$; and $\boldsymbol{j}_{-}^{(2)}(x, \lambda)$ if $\Im \lambda<0$. For the "." copy we define $\boldsymbol{j}(x, Q)$ to be $\boldsymbol{j}_{-}^{(1)}(x, \lambda)$ if $\Im \lambda>0$; and $\boldsymbol{j}_{+}^{(1)}(x, \lambda)$ if $\Im \lambda<0$. In the vicinity of $P_{ \pm}$ the function $\boldsymbol{j}(x, Q)$ has asymptotics,

$$
\boldsymbol{j}(x, Q)=e^{ \pm i \frac{\lambda}{2} x}\left[\boldsymbol{j}_{0} / \hat{\boldsymbol{j}}_{0}+o(1)\right],
$$

where $\lambda=\lambda(Q)$ and

$$
\boldsymbol{j}_{0}=\left[\begin{array}{l}
0 \\
1
\end{array}\right] \quad \hat{\boldsymbol{j}}_{0}=\left[\begin{array}{l}
1 \\
0
\end{array}\right] .
$$

Therefore, $\boldsymbol{j}(x, Q)$ can be viewed as a BA function for the singular curve $\Gamma_{\infty}$.

We also introduce the matrix BA function

$$
H_{+}(\lambda)=\left[\boldsymbol{j}_{-}^{(1)}(\lambda), \boldsymbol{j}_{+}^{(2)}(\lambda)\right] \quad \text { and } \quad H_{-}(\lambda)=\left[\boldsymbol{j}_{+}^{(1)}(\lambda), \boldsymbol{j}_{-}^{(2)}(\lambda)\right]
$$

analytic in the upper/lower half-plane respectively. They are connected by the gluing condition

$$
H_{-}(x, \lambda)=H_{+}(x, \lambda) S(\lambda), \quad \text { where } \quad \lambda \in \mathbb{R}
$$

and the scattering matrix $S(\lambda)$

$$
S(\lambda)=\frac{1}{a}\left[\begin{array}{cc}
1 & \bar{b} \\
-b & 1
\end{array}\right]
$$

The adjoint (dual) Jost solution $\boldsymbol{j}^{+}$at the point $Q$ is defined by the formula

$$
\boldsymbol{j}^{+}(x, Q) \equiv \boldsymbol{j}(x, Q)^{T} .
$$

Any Jost solution satisfies $\left[J \partial_{x}-J V\right] \boldsymbol{j}=0$. By analogy with the periodic case one can prove that $\boldsymbol{j}^{+}$satisfies $\boldsymbol{j}^{+}\left[J \partial_{x}-J V\right]=0$.

The matrices $H_{+}^{+}$and $H_{-}^{+}$are defined as

$$
H_{+}^{+}(\lambda)=\sigma_{1} H_{+}^{T}(\lambda)=\left[\begin{array}{l}
\boldsymbol{j}_{+}^{(2) T} \\
\boldsymbol{j}_{-}^{(1) T}
\end{array}\right], \quad H_{-}^{+}(\lambda)=\sigma_{1} H_{-}^{T}(\lambda)=\left[\begin{array}{l}
\boldsymbol{j}_{-}^{(2) T} \\
\boldsymbol{j}_{+}^{(1) T}
\end{array}\right] .
$$

Extending $a$ into the lower half-plane by the formula $a^{*}(\lambda)=\overline{a(\bar{\lambda})}$, we define

$$
H_{+}^{*}(\lambda)=-\frac{\sigma_{3}}{a(\lambda)} H_{+}^{+}(\lambda) \quad \text { for } \quad \Im \lambda>0
$$

and

$$
H_{-}^{*}(\lambda)=-\frac{\sigma_{3}}{a^{*}(\lambda)} H_{-}^{+}(\lambda) \quad \text { for } \quad \Im \lambda<0 .
$$

It is easy to check that the dual gluing condition holds

$$
H_{-}^{*}(x, \lambda)=S^{-1}(\lambda) H_{+}^{*}(x, \lambda), \quad \text { where } \quad \lambda \in \mathbb{R}
$$


and

$$
S^{-1}(\lambda)=\frac{1}{a^{*}}\left[\begin{array}{cc}
1 & -\bar{b} \\
b & 1
\end{array}\right]
$$

Next two lemmas state asymptotic properties of Jost solutions which will be used in computations with symplectic forms.

Lemma 3.1. (i) For fixed $x$ the following formulas hold

$$
\boldsymbol{j}_{+}^{(2)}(x, \lambda)=e^{+i \frac{\lambda}{2} x} \sum_{s=0}^{\infty} \boldsymbol{j}_{s}(x) \lambda^{-s}=e^{+i \frac{\lambda}{2} x} \sum_{s=0}^{\infty}\left[\begin{array}{l}
g_{s} \\
k_{s}
\end{array}\right] \lambda^{-s},
$$

where $g_{0}=0, \quad k_{0}=1$, and $d^{10}$

$$
\boldsymbol{j}_{-}^{(1)}(x, \lambda)=e^{-i \frac{\lambda}{2} x} \sum_{s=0}^{\infty} \hat{\boldsymbol{j}}_{s}(x) \lambda^{-s}=e^{-i \frac{\lambda}{2} x} \sum_{s=0}^{\infty}\left[\begin{array}{l}
\hat{k}_{s} \\
\hat{g}_{s}
\end{array}\right] \lambda^{-s},
$$

where $\hat{g}_{0}=0, \quad \hat{k}_{0}=1$. The expansion has an asymptotic character for $\lambda: \delta \leq$ $\arg \lambda \leq \pi-\delta, \delta>0$.

(ii) The coefficients $g_{1}, k_{1}$ are given by the formulas

$$
g_{1}=-i \bar{\psi}, \quad k_{1}=i \int_{x}^{+\infty}\left|\psi\left(x^{\prime}\right)\right|^{2} d x^{\prime}
$$

and

$$
\hat{g}_{1}=i \psi, \quad \hat{k}_{1}=i \int_{-\infty}^{x}\left|\psi\left(x^{\prime}\right)\right|^{2} d x^{\prime}
$$

Proof (i). Using $\left.{ }^{11} \partial_{\xi}^{n} \Gamma_{+}^{(2)}(x, \xi)\right|_{\xi=\infty}=0$, for $n=0,1, \ldots$ and integrating $n$ times by parts,

$$
\begin{aligned}
\boldsymbol{j}_{+}^{(2)}(x, \lambda)= & e^{\frac{i \lambda x}{2}}\left[\begin{array}{l}
0 \\
1
\end{array}\right]+\int_{x}^{\infty} \Gamma_{+}^{(2)}(x, \xi) e^{\frac{i \lambda \xi}{2}} d \xi \\
\ldots= & e^{\frac{i \lambda x}{2}}\left[\begin{array}{l}
0 \\
1
\end{array}\right]-\frac{e^{\frac{i \lambda x}{2}}}{\left(\frac{i \lambda}{2}\right)} \Gamma_{+}^{(2)}(x, x)+\frac{e^{\frac{i \lambda x}{2}}}{\left(\frac{i \lambda}{2}\right)^{2}} \partial_{\xi} \Gamma_{+}^{(2)}(x, x)-\ldots \\
& +(-1)^{n} \frac{e^{\frac{i \lambda x}{2}}}{\left(\frac{i \lambda}{2}\right)^{n}} \partial_{\xi}^{n-1} \Gamma_{+}^{(2)}(x, x)+(-1)^{n} \frac{1}{\left(\frac{i \lambda}{2}\right)^{n}} \int_{x}^{\infty} \partial_{\xi}^{n} \Gamma_{+}^{(2)}(x, \xi) e^{\frac{i \lambda \xi}{2}} d \xi .
\end{aligned}
$$

This implies the existence and asymptotic character of the expansion in the parameter $\lambda$. The other infinity can be treated similarly.

\footnotetext{
${ }^{10}$ Operation $`$ applied to a scalar signifies complex conjugation and reversal of infinities, see formulas in the part (ii) below.

${ }^{11} \Gamma=\left[\Gamma^{(1)}, \Gamma^{(2)}\right]$.
} 
(ii). Consider $\boldsymbol{j}_{+}^{(2)}(x, \lambda)$ first. The differential equation $\boldsymbol{j}^{\prime}=V \boldsymbol{j}$ implies

$$
-\left[\begin{array}{l}
g_{s}^{\prime} \\
k_{s}^{\prime}
\end{array}\right]+Y_{0}\left[\begin{array}{c}
g_{s} \\
k_{s}
\end{array}\right]=\frac{i}{2}\left(I+\sigma_{3}\right)\left[\begin{array}{c}
g_{s+1} \\
k_{s+1}
\end{array}\right], \quad s=0,1, \ldots ;
$$

and $g_{0}=0, \quad k_{0}=1$.

This recurrent relation leads to the identities

$$
\begin{aligned}
& -g_{s}^{\prime}+\bar{\psi} k_{s}=i g_{s+1}, \\
& -k_{s}^{\prime}+\psi g_{s}=0 .
\end{aligned}
$$

For $s \geq 1$ we have the boundary condition

$$
\left.g_{s}(x)\right|_{x=+\infty}=\left.k_{s}(x)\right|_{x=+\infty}=0 .
$$

These imply the stated formulas for $g_{1}, k_{1}$.

For $\boldsymbol{j}_{-}^{(1)}(x, \lambda)$ the differential equation implies

$$
-\left[\begin{array}{c}
k_{s}^{\prime} \\
g_{s}^{\prime}
\end{array}\right]+Y_{0}\left[\begin{array}{l}
k_{s} \\
g_{s}
\end{array}\right]=\frac{i}{2}\left(-I+\sigma_{3}\right)\left[\begin{array}{c}
k_{s+1} \\
g_{s+1}
\end{array}\right], \quad s=0,1, \ldots ;
$$

and $k_{0}=1, \quad g_{0}=0$.

The recurrent relation produces the identities

$$
\begin{aligned}
& -g_{s}^{\prime}+\psi k_{s}=-i g_{s+1}, \\
& -k_{s}^{\prime}+\bar{\psi} g_{s}=0 .
\end{aligned}
$$

For $s \geq 1$ we have the boundary condition

$$
\left.g_{s}(x)\right|_{x=-\infty}=\left.k_{s}(x)\right|_{x=-\infty}=0 .
$$

These imply the stated formulas for $\hat{g}_{1}, \hat{k}_{1}$. We are done.

Remark. It is interesting to compare asymptotic expansions for $\boldsymbol{j}_{-}^{(1)} / \boldsymbol{j}_{+}^{(2)}$ and $\boldsymbol{e}(x, Q)$. For the Jost solution $\boldsymbol{j}_{-}^{(1)}(x, \lambda)$ normalized at the left

$$
\boldsymbol{j}_{-}^{(1)}(x, \lambda)=e^{-\frac{i \lambda x}{2}}\left(\left[\begin{array}{l}
1 \\
0
\end{array}\right]+\frac{1}{\lambda}\left[\begin{array}{c}
i \int_{-\infty}^{x}|\psi|^{2} \\
i \psi
\end{array}\right]+\ldots\right)
$$

and

$$
e^{i \frac{\lambda}{2} l} \boldsymbol{e}(x, Q)=e^{-\frac{i \lambda x}{2}}\left(\left[\begin{array}{l}
1 \\
0
\end{array}\right]+\frac{1}{\lambda}\left[\begin{array}{c}
-i \psi_{0}+i \int_{-l}^{x}|\psi|^{2} \\
i \psi
\end{array}\right]+\ldots\right), \quad Q \in\left(P_{-}\right) .
$$

If $\psi$ is compactly supported and $l$ becomes sufficiently large, then $e^{i \frac{\lambda}{2} l} \boldsymbol{e}(x, Q)=$ $\boldsymbol{j}(x, \lambda)$. For the Jost solution normalized at the right the situation is slightly different. If one defines the new $\boldsymbol{e}(x, Q) \equiv \boldsymbol{e}(x, Q) w^{-1}(Q)$ which is the Floquet solution normalized at the right end $x=l$ of the interval, then for compactly supported $\psi$ and sufficiently large $l$ the new $e^{-i \frac{\lambda}{2} l} \boldsymbol{e}(x, Q)=\boldsymbol{j}_{+}^{(2)}(x, \lambda)$.

A result similar to Lemma 4.1 holds for Jost solutions analytic in the lower half-plane. 
Lemma 3.2. (i) For fixed $x$ the following formulas hold

$$
\boldsymbol{j}_{+}^{(1)}(x, \lambda)=e^{-i \frac{\lambda}{2} x} \sum_{s=0}^{\infty} \boldsymbol{j}_{s}(x) \lambda^{-s}=e^{-i \frac{\lambda}{2} x} \sum_{s=0}^{\infty}\left[\begin{array}{c}
h_{s} \\
f_{s}
\end{array}\right] \lambda^{-s},
$$

where $h_{0}=1, \quad f_{0}=0$, and

$$
\boldsymbol{j}_{-}^{(2)}(x, \lambda)=e^{+i \frac{\lambda}{2} x} \sum_{s=0}^{\infty} \hat{\boldsymbol{j}}_{s}(x) \lambda^{-s}=e^{+i \frac{\lambda}{2} x} \sum_{s=0}^{\infty}\left[\begin{array}{l}
\hat{f}_{s} \\
\hat{h}_{s}
\end{array}\right] \lambda^{-s},
$$

where $\hat{h}_{0}=1, \quad \hat{f}_{0}=0$. The expansion has an asymptotic character for $\lambda$ : $-\delta \geq \arg \lambda \geq-\pi+\delta, \delta>0$.

(ii) The coefficients $h_{1}, f_{1}$ are given by the formulas

$$
f_{1}=i \psi, \quad h_{1}=-i \int_{x}^{+\infty}\left|\psi\left(x^{\prime}\right)\right|^{2} d x^{\prime}
$$

and

$$
\hat{f}_{1}=-i \bar{\psi}, \quad \hat{h}_{1}=-i \int_{-\infty}^{x}\left|\psi\left(x^{\prime}\right)\right|^{2} d x^{\prime}
$$

Similar to the periodic case we will need time dependent BA functions. They are obtained by an elementary construction.

Lemma 3.3. [7]. There exists the Jost solution $\boldsymbol{j}(\tau, x, t, Q)$ on the curve $\Gamma_{\infty}$ with three time parameters $\tau, x$ and $t$ which satisfies the differential equations:

$$
\begin{aligned}
& {\left[\partial_{\tau}-V_{1}(\tau, x, t)\right] \boldsymbol{j}(\tau, x, t, Q)=0,} \\
& {\left[\partial_{x}-V_{2}(\tau, x, t)\right] \boldsymbol{j}(\tau, x, t, Q)=0,} \\
& {\left[\partial_{t}-V_{3}(\tau, x, t)\right] \boldsymbol{j}(\tau, x, t, Q)=0 .}
\end{aligned}
$$

Proof. We consider "+" sheet and the upper half-plane where $\boldsymbol{j}(x, Q)=\boldsymbol{j}_{+}^{(2)}(x, \lambda)$. First, we construct $\boldsymbol{j}(\tau, x, t, Q)$ such that $\left[\partial_{x}-V_{2}(\tau, x, t)\right] \boldsymbol{j}(\tau, x, t, Q)=0$ normalized for all $\tau$ and $t$ as

$$
\boldsymbol{j}(\tau, x, t, Q) \sim e^{+i \frac{\lambda}{2} x}\left(\left[\begin{array}{l}
0 \\
1
\end{array}\right]+o(1)\right), \quad \text { when } \quad x \rightarrow \infty .
$$

Then, we construct $\boldsymbol{j}\left(\tau, x, t, \epsilon_{ \pm} Q\right)$ on the lower sheet as a solution

$$
\left[\partial_{x}-V_{2}(\tau, x, t)\right] \boldsymbol{j}\left(\tau, x, t, \epsilon_{ \pm} Q\right)=0
$$

for the same value of the spectral parameter $\lambda=\lambda(Q)$ normalized for all $\tau$ and $t$ as

$$
\boldsymbol{j}\left(\tau, x, t, \epsilon_{ \pm} Q\right) \sim e^{-i \frac{\lambda}{2} x}\left(\left[\begin{array}{l}
1 \\
0
\end{array}\right]+o(1)\right), \quad \text { when } \quad x \rightarrow-\infty
$$


The solutions $\boldsymbol{j}(\tau, x, t, Q)$ and $\boldsymbol{j}\left(\tau, x, t, \epsilon_{ \pm} Q\right)$ span the kernel of the operator $\left[\partial_{x}-\right.$ $\left.V_{2}(\tau, x, t)\right]$. Now we introduce

$$
\boldsymbol{j}_{\text {new }}(\tau, x, t, Q) \equiv e^{-i \frac{1}{2} \tau-i \frac{\lambda^{2}}{2} t} \boldsymbol{j}(\tau, x, t, Q)
$$

which is the desired solution. Evidently,

$$
\left[\partial_{x}-V_{2}(\tau, x, t)\right] \boldsymbol{j}_{\text {new }}(\tau, x, t, Q)=0 .
$$

To prove the first identity of the statement we note, that commutativity of the operators $\partial_{\tau}-V_{1}$ and $\partial_{x}-V_{2}$ implies

$$
\left[\partial_{\tau}-V_{1}(\tau, x, t)\right] \boldsymbol{j}_{\text {new }}(\tau, x, t, Q)=c_{1}(\tau, t) \boldsymbol{j}(\tau, x, t, Q)+c_{2}(\tau, t) \boldsymbol{j}\left(\tau, x, t, \epsilon_{ \pm} Q\right) .
$$

From another side as $x \rightarrow+\infty$,

$\left[\partial_{\tau}-V_{1}(\tau, x, t)\right] \boldsymbol{j}_{\text {new }}(\tau, x, t, Q)=\left[\partial_{\tau}-\frac{i}{2} \sigma_{3}\right] e^{-i \frac{1}{2} \tau+i \frac{\lambda}{2} x-i \frac{\lambda^{2}}{2} t}\left(\left[\begin{array}{l}0 \\ 1\end{array}\right]+o(1)\right)=o(1)$.

Due to the linear independence of the solutions $\boldsymbol{j}(\tau, x, t, Q)$ and $\boldsymbol{j}\left(\tau, x, t, \epsilon_{ \pm} Q\right)$ we have $c_{1}(\tau, t)=c_{2}(\tau, t)=0$.

Similarly it can be proved that

$$
\left[\partial_{t}-V_{3}(\tau, x, t)\right] \boldsymbol{j}_{\text {new }}(\tau, x, t, Q)=0 .
$$

Another sheet of $\Gamma_{\infty}$ can be treated the same way. We are done.

Remark. It is easy to see that,

$$
\boldsymbol{j}_{\text {new }}(\tau, x, t, Q)=e^{ \pm i\left(-\frac{1}{2} \tau+\frac{\lambda}{2} x-\frac{\lambda^{2}}{2} t\right)}\left[\boldsymbol{j}_{0} / \hat{\boldsymbol{j}}_{0}+o(1)\right] \quad Q \in\left(P_{ \pm}\right) .
$$

Thus the standard Jost solution with asymtotics 3.5 can be obtained from the BA functions if one puts $\tau$ and $t$ equal to 0 .

3.2. The symplectic structures. We are ready to introduce the scattering version of the Krichever-Phong formula. The everaging is defined now as an integral over the entire line $<\bullet>=\int_{-\infty}^{+\infty} d x$.

Theorem 3.4. The formula

$$
\omega_{0}=\text { trace res } \frac{1}{2}\left[<H_{+}^{*} J \delta V \wedge \delta H_{+}>+<H_{-}^{*} J \delta V \wedge \delta H_{-}>\right] d \lambda,
$$

defines a closed 2-form $\omega_{0}$ on the space of operators $\partial_{x}-V_{2}$ with potential from the Schwartz class $S(\mathbb{R})$. The flows $e^{t X_{m}}, m=1,2, \ldots$ on the space of operators defined by the formula

$$
\left[\partial_{\tau_{m}}-V_{m}, \partial_{x}-V_{2}\right]=0
$$

are Hamiltonian with respect to the 2 -form $\omega_{0}$ with Hamiltonian function $H_{m}$ (up to a non-essential constant factor). 
Remark 1. The symbol

$$
\text { res }<H_{+}^{*} J \delta V \wedge \delta H_{+}>
$$

means the coefficient corresponding to the term $\frac{1}{\lambda}$ in the power series expansion near infinity in the upper half-plane. The second term

$$
\text { res }<H_{-}^{*} J \delta V \wedge \delta H_{-}>
$$

is defined in the same way, only the upper half-plane plane is replaced with the lower half-plane.

Remark 2. The formula

$$
\omega_{n}=\text { trace res } \frac{\lambda^{n}}{2}\left[<H_{+}^{*} J \delta V \wedge \delta H_{+}>+<H_{-}^{*} J \delta V \wedge \delta H_{-}>\right] d \lambda,
$$

where $n=0,1 \ldots$, defines a closed 2 -forms $\omega_{n}$ on the space of operators $\partial_{x}-V_{2}$ with potential from the Schwartz class $S(\mathbb{R})$ that satisfy the constraints $H_{k}=$ const, $k=1, \ldots, n$. It is instructive to compute explicitly the symplectic forms for small $n$. The first few are given by the formulas

$$
\omega_{0}=2 i<\delta \bar{\psi} \wedge \delta \psi>
$$

and

$$
\omega_{1}=<\delta \psi \wedge \delta \bar{\psi}^{\prime}+\delta \bar{\psi} \wedge \delta \psi^{\prime}+\delta\left[\int_{-\infty}^{x}|\psi|^{2}-\int_{x}^{\infty}|\psi|^{2}\right] \wedge \delta|\psi|^{2}>.
$$

subject to the constrain $H_{1}=$ const. The derivation employs Lemmas 3.1-3.2 and similar to the periodic case.

Proof. Closeness of the form $\omega_{0}$ follows either from the explicit formula or from the result of the next theorem. The proof of the second statement we present for the first $e^{t X_{1}}$ flow. The time dependent Jost solutions entering into 3.9 are constructed in Lemma 3.4. Let $i_{\partial_{t}}$ be the construction operator produced by the vector field $X_{1}$. We will prove $i_{\partial_{t}} \omega_{0}=-\delta 2 H_{1}$. For the first term in 3.8

trace $\quad$ res $<H_{+}^{*} J \delta V \wedge \delta H_{+}>d \lambda$

$$
=\operatorname{res} \frac{1}{a}<\boldsymbol{j}_{-}^{(1) T} J \delta V \wedge \delta \boldsymbol{j}_{+}^{(2)}>d \lambda-\operatorname{res} \frac{1}{a}<\boldsymbol{j}_{+}^{(2) T} J \delta V \wedge \delta \boldsymbol{j}_{-}^{(1)}>d X(3.9)
$$

Applying the contraction operator to the first term in 3.9

$$
\begin{aligned}
i_{\partial_{t}} \operatorname{res} \frac{1}{a}< & \boldsymbol{j}_{-}^{(1) T} J \delta V \wedge \delta \boldsymbol{j}_{+}^{(2)}>d \lambda \\
& =\operatorname{res} \frac{1}{a}<\boldsymbol{j}_{-}^{(1) T} J V^{\bullet} \delta \boldsymbol{j}_{+}^{(2)}>d \lambda-\operatorname{res} \frac{1}{a}<\boldsymbol{j}_{-}^{(1) T} J \delta V \boldsymbol{j}_{+}^{(2) \bullet}>d \lambda .
\end{aligned}
$$

From 3.3 we have

$$
\frac{1}{a}=a_{0}+\frac{a_{1}}{\lambda}+\ldots, \quad \text { where } \quad a_{0}=1, \quad a_{1}=-i \int_{-\infty}^{+\infty}|\psi|^{2} .
$$


Using $V^{\bullet}=\left[\frac{i}{2} \sigma_{3}, V\right]=i \sigma_{3} V$, and Lemma 3.1, we have

$$
\operatorname{res} \frac{1}{a}<\boldsymbol{j}_{-}^{(1) T} J V^{\bullet} \delta \boldsymbol{j}_{+}^{(2)}>d \lambda=a_{0}<\hat{\boldsymbol{j}}_{0}^{T} J i \sigma_{3} V \delta \boldsymbol{j}_{1}>=-<\psi \delta \bar{\psi}>.
$$

Similarly, using $\boldsymbol{j}^{\bullet}=\frac{i}{2} \sigma_{3} \boldsymbol{j}$, we have

$$
\begin{aligned}
\operatorname{res} \frac{1}{a}<\boldsymbol{j}_{-}^{(1) T} J \delta V \boldsymbol{j}_{+}^{(2) \bullet}>d \lambda & =a_{1}<\hat{\boldsymbol{j}}_{0}^{T} J \delta V \frac{i}{2} \sigma_{3} \boldsymbol{j}_{0}> \\
& +a_{0}\left[<\hat{\boldsymbol{j}}_{0}^{T} J \delta V \frac{i}{2} \sigma_{3} \boldsymbol{j}_{1}>+<\hat{\boldsymbol{j}}_{1}^{T} J \delta V \frac{i}{2} \sigma_{3} \boldsymbol{j}_{0}>\right] .
\end{aligned}
$$

The first term vanishes, the second produces

$$
=\frac{1}{2}<\bar{\psi} \delta \psi-\psi \delta \bar{\psi}>\text {. }
$$

Finally,

$$
i_{\partial_{t}} \operatorname{res} \frac{1}{a}<\boldsymbol{j}_{-}^{(1) T} J \delta V \wedge \delta \boldsymbol{j}_{+}^{(2)}>d \lambda=-\delta H_{1} .
$$

The second term in formula 3.9 can be treated similarly. Therefore,

$$
i_{\partial_{t}} \text { trace res }<H_{+}^{*} J \delta V \wedge \delta H_{+}>=-2 \delta H_{1} \text {. }
$$

The second term in formula 3.8 produces the same result. The proof is finished.

3.3. Action-angle variables. In response to infinitesimal deformations of the matrix $\tilde{V}=V+\delta V$ the matrix $T(\lambda)$ changes according to the rule: $\tilde{T}(\lambda)=$ $T(\lambda)+\delta T(\lambda)+\ldots$ The next result is similar to Lemma 2.3 of the periodic case.

Lemma 3.5. The following formula holds

$$
<H_{+}^{+} J \delta V H_{+}>=\left[\begin{array}{cc}
-\delta a & \bar{b} \delta a-a \delta \bar{b} \\
a \delta b-b \delta a & -\delta a
\end{array}\right],
$$

with averaging defined as

$$
<H_{+}^{+} J \delta V H_{+}>=\int_{-\infty}^{+\infty} H_{+}^{+}(x, \lambda) J \delta V(x) H_{+}(x, \lambda) d x .
$$

Proof. Let us assume, first, that $\psi$ has compact support. We denote by $\tilde{V}, \tilde{T}$ and $\tilde{j}$ deformed matrices $V, T$ and the Jost solution $\boldsymbol{j}$. We will derive the expression for $\left\langle\boldsymbol{j}_{+}^{(2) T} J \delta V \boldsymbol{j}_{+}^{(1)}>\right.$ in the left-upper corner. First, we obtain the formula

$$
<\boldsymbol{j}^{+} J \delta V \boldsymbol{j}>+ \text { lower order terms }=\left.\boldsymbol{j}^{+} J \tilde{\boldsymbol{j}}\right|_{-L} ^{+L} .
$$

Indeed,

$$
\begin{aligned}
\boldsymbol{j}^{+}\left(\left[J \partial_{x}-J \tilde{V}\right] \tilde{\boldsymbol{j}}\right) & =0 \\
\left(\boldsymbol{j}^{+}\left[J \partial_{x}-J V\right]\right) \tilde{\boldsymbol{j}} & =0 .
\end{aligned}
$$


Subtracting one identity from another we have

$$
\boldsymbol{j}^{+} J \delta V \boldsymbol{j}+\text { lower order terms }=\boldsymbol{j}^{+}(J \partial \tilde{\boldsymbol{j}})-\left(\boldsymbol{j}^{+} J \partial\right) \tilde{\boldsymbol{j}} \text {. }
$$

Integrating the RHS in $\mathrm{x}$ variable we obtain

$$
\int_{-L}^{+L}\left[\boldsymbol{j}^{+} J \tilde{\boldsymbol{j}}^{\prime}+\boldsymbol{j}^{+\prime} J \tilde{\boldsymbol{j}}\right] d x=\left.\boldsymbol{j}^{+} J \tilde{\boldsymbol{j}}\right|_{-L} ^{+L} .
$$

This implies 3.10. Now using formulas for the asymptotics of $\boldsymbol{j}_{+}^{(2)}$ and $\boldsymbol{j}_{-}^{(1)}$ for the RHS of 3.10, we have

$$
\begin{aligned}
\left.\boldsymbol{j}_{+}^{(2) T} J \tilde{\boldsymbol{j}}_{-}^{(1)}\right|_{-L} ^{+L} & =\left.\boldsymbol{f}_{\rightarrow}^{T} J\left[\tilde{a} \boldsymbol{f}_{\leftarrow}+\tilde{b} \boldsymbol{f}_{\rightarrow}\right]\right|^{+L}-\left.\left[a \boldsymbol{f}_{\rightarrow}^{T}-\bar{b} \boldsymbol{f}_{\leftarrow}^{T}\right] J \boldsymbol{f}_{\leftarrow}\right|_{-L} \\
& =\tilde{a} \boldsymbol{f}_{\rightarrow}^{T} J \boldsymbol{f}_{\leftarrow}-a \boldsymbol{f}_{\rightarrow}^{T} J \boldsymbol{f}_{\leftarrow}=a-\tilde{a}=-\delta a+\text { lower order terms. }
\end{aligned}
$$

Collecting terms of the same order, we obtain the result. The case of a potential with non-compact support can be considered using approximation arguments. For other entries the arguments are the same. Lemma is proved.

Theorem 3.6. The following formulas hold

$$
\omega_{0}=\frac{1}{\pi i} \int_{-\infty}^{+\infty} \frac{\delta \bar{b}(\lambda) \wedge \delta b(\lambda)}{|a(\lambda)|^{2}} d \lambda
$$

Proof. By the Cauchy integral formula

$$
\frac{1}{2} \text { trace res }<H_{+}^{*} J \delta V \wedge \delta H_{+}>=-\frac{1}{2 \pi i} \int_{-\infty}^{+\infty} \operatorname{trace}<H_{+}^{*} J \delta V \wedge \delta H_{+}>d \lambda,
$$

and

$$
\frac{1}{2} \text { trace res }<H_{-}^{*} J \delta V \wedge \delta H_{-}>=\frac{1}{2 \pi i} \int_{-\infty}^{+\infty} \operatorname{trace}<H_{-}^{*} J \delta V \wedge \delta H_{-}>d \lambda .
$$

Taking sum

$$
\omega_{0}=\frac{1}{2 \pi i} \int_{-\infty}^{+\infty} \operatorname{trace}<H_{-}^{*} J \delta V \wedge \delta H_{-}>d \lambda-\frac{1}{2 \pi i} \int_{-\infty}^{+\infty} \operatorname{trace}<H_{+}^{*} J \delta V \wedge \delta H_{+}>d \lambda .
$$

Using 3.6, 3.7

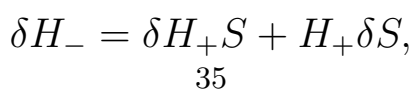


we obtain

$$
\begin{aligned}
\omega_{0}= & \frac{1}{2 \pi i} \int_{-\infty}^{+\infty} \operatorname{trace} S^{-1}<H_{+}^{*} J \delta V \wedge \delta H_{+}>S d \lambda+\int_{-\infty}^{+\infty} \operatorname{trace} S^{-1}<H_{+}^{*} J \delta V H_{+}>\wedge \delta S d \lambda \\
& -\frac{1}{2 \pi i} \int_{-\infty}^{+\infty} \operatorname{trace}<H_{+}^{*} J \delta V \wedge \delta H_{+}>d \lambda \\
= & \int_{-\infty}^{+\infty} \operatorname{trace}<H_{+}^{*} J \delta V H_{+}>\wedge \delta S S^{-1} d \lambda .
\end{aligned}
$$

Now, applying the result of Lemma 3.5, we have

$$
\omega_{0}=\frac{1}{2 \pi i} \int_{-\infty}^{+\infty} \operatorname{trace}-\frac{\sigma_{3}}{a^{*}}\left[\begin{array}{cc}
-\delta a & \bar{b} \delta a-a \delta \bar{b} \\
a \delta b-b \delta a & -\delta a
\end{array}\right] \wedge \delta S S^{-1} d \lambda .
$$

After simple algebra we arrive at the stated identity. Theorem is proved.

Remark 1. The formula of Theorem can be put easily into more familiar form using the identities

$$
|a|^{2}-|b|^{2}=1, \quad \delta \log |b|^{2}=\frac{2|a| \delta|a|}{|a|^{2}-1} .
$$

Indeed,

$$
\begin{aligned}
\frac{1}{i} \frac{\delta \bar{b}(\lambda) \wedge \delta b(\lambda)}{|a(\lambda)|^{2}} & =\frac{|b(\lambda)|^{2} \delta \log |b(\lambda)|^{2} \wedge \delta \operatorname{ph} b(\lambda)}{|a(\lambda)|^{2}} \\
& =2 \delta \log |a(\lambda)| \wedge \delta \operatorname{ph} b(\lambda)
\end{aligned}
$$

Therefore,

$$
\omega_{0}=\frac{1}{\pi i} \int_{-\infty}^{+\infty} \frac{\delta \bar{b}(\lambda) \wedge \delta b(\lambda)}{|a(\lambda)|^{2}}=\frac{2}{\pi} \int_{-\infty}^{+\infty} \delta \log |a(\lambda)| \wedge \delta \operatorname{ph} b(\lambda) d \lambda .
$$

Remark 2. The formula

$$
\omega_{n}=\frac{1}{\pi i} \int_{-\infty}^{+\infty} \frac{\delta \bar{b}(\lambda) \wedge \delta b(\lambda)}{|a(\lambda)|^{2}} \lambda^{n} d \lambda, \quad n=1,2, \ldots
$$

subject to the constrains $H_{k}=$ const, $k=1, \ldots, n$; gives Darboux coordinates for higher symplectic forms. 


\section{REFERENCES}

[1] V.I. Arnold Mathematical methods of classical mechanics. Springer-Verlag, 1978.

[2] R.F. Bikbaev and S.B. Kuksin On the parametrization of finite-gap solutions by frequency vector and wavenumber vector and a theorem of I. Krichever Lett. Math. Phys. 166, 1994, pp. 115-122.

[3] Eric D'Hooker, I.M. Krichever and D.H Phong Seiberg-Witten Theory, Symplectic Forms, and Hamiltonian Theory of Solitons, Beijing and Hangzhou 2002; preprint hep-th 0212313

[4] L.D. Faddeev and L.A. Takhtadzian Hamiltonian Methods in the Theory of Solitons. Springer-Verlag, 1987.

[5] L.D. Faddeev and V.E. Zakharov Korteweg-de Vries equation: A completely integrable Hamiltonian system. Funk. Analiz and its Appl. Vol 5, 1971, 18-27.

[6] H. Flashka and D. McLaughlin Canonically conjugate variables for the Korteweg-de Vries equation and the Toda lattice with the periodic boundary conditions Progr. Theoret. Phys. 55 (1976) no 2, 438-456.

[7] A.R. Its The Liouville theorem and the inverse scattering method. Journ. of Soviet Math. 31 (1985), 6, 299-334.

[8] M.G. Krein On the theory of accelerant and S-matrices of canonical differential equations. Dokl. Akad. Nauk. SSSR 111 1956, 1167-1170.

[9] M.G. Krein and P.E. Melik-Adamian A contribution to the theory of S-matrices of canonical differential equations with summable potential. Dokl. Akad. Nauk. Armjan. SSR 46 $1968,150-155$.

[10] I. M. Krichever Methods of algebraic geometry in the theory of nonlinear equations Uspehi Mat. Nauk 32, (1977), no 6, 183-208.

[11] I. M. Krichever Elliptic solutions to the difference nonlinear equations and nested Bethe ansatz equations, Calogero-Moser-Sutherland models (Montreal, QC,1997) CRM Ser. Math. Phys. Spinger, New York, 2000.

[12] I. M. Krichever Perturbation Theory in Periodic Problems for two Dimensional Integrable Systems Sov. Sci. Rev. C. Math. Phys. Vol. 9, 1992, pp 1-103.

[13] I.M. Krichever and D.H Phong Symplectic forms in the theory of solitons, Surveys in Differential Geometry: Integrable Systems, v.4 (1999), International Press, Boston, 1999; preprint hep-th 9708170

[14] P.P. Kulish and A.G. Reiman Hierarchy of Symplectic Forms for the Schrodinger and Dirac Equations on a line. Zap. LOMI, vol 77, 134-147, 1978.

[15] V.B. Kuznetzov, F.W. Nijhoff, E.K. Sklyanin Separation of variables for the Ruijsenaars system CMP, 189 (1997), no 3, 855-877.

[16] P.E. Melik-Adamian On the properties of $S$-matrices of canonical differential equations on the entire line. Dokl. Akad. Nauk. Armjan. SSR 58 1974, 199-205

[17] H.P. McKean Trace Formulas and the Canonical 1-form. Algebraic Aspects of Integrable Systems. Progr. Nonlinear Differential Equations, 26; Birkhauser Boston, MA, 1997, 217235.

[18] H.P. McKean A Novel Aspect of Action-Angle Variables. Proceedings of Symp in Pure Mathematics. vol 65, 1999, 155-160.

[19] H.P. McKean and K.L. Vaninsky Action-angle variables for the Cubic Schrodinger Equation. CPAM, vol L, 1997, 489-562.

[20] J. Moser Integrable Hamiltonian systems and spectral theory Lezioni Fermiane. Scuola Normale Superiore, Piza, 1983; 
[21] S.P. Novikov, S.V. Manakov, L.P. Pitaevsky Theory of Solitons. The inverse scattering method. Trans from Russian, Consultants Bureau [Plenum], New York, 1984, 276 pp.

[22] S.P. Novikov and A.P. Veselov Poisson brackets and complex tori. Trudy Math, Inst Steklov, 165 (1984), 49-61.

[23] N. Seiberg and E. Witten Electro-magnetic duality, monopole condensation, and confinement in $N=2$ supersymmetric Yang-Mills theory, Nucl. Phys. B 426 (1994) 19-53, hepth/9407087;

[24] N. Seiberg and E. Witten, Monopoles, duality, and chiral symmetry breaking in $N=2$ supersymmetric QCD, Nucl. Phys. B 431 (1994) 494, hep-th/9410167.

[25] E.K. Sklyanin Separation of variables - new trends. Quantum field theory, integrable models and beyond (Kyoto, 1994). Progr Theor Phys. Suppl. n 118 (1995) 35-60.

[26] K.L. Vaninsky A convexity theorem in the Scattering theory for the Dirac operator. TAMS, 350, 1998, 1895-1911.

[27] K.L. Vaninsky The scattering divisor for the cubic Schrödinger equation. paper in preparation.

[28] V.E. Zakharov and S.V. Manakov On the complete integrability of a nonlinear Schrodinger Equation. Teor. and Math Phys, vol 19, 332-343, 1974. 